\title{
Reduced order model of a two-phase loop thermosyphon by modal identification method
}

Serge BODJONA, Manuel GIRAULT, Etienne VIDECOQ, Yves BERTIN

Institut Pprime, CNRS - ENSMA - Université de Poitiers

Département Fluides, Thermique, Combustion

ENSMA, Téléport 2, 1, avenue Clément Ader

BP 40109, F86961 FUTUROSCOPE CHASSENEUIL Cedex

Corresponding author: etienne.videcoq@ensma.fr, Phone number : +335494981 17

\begin{abstract}
This study focuses on the model reduction of a two-phase loop thermosyphon. The aim is to propose a nonlinear reduced order model able to mimic the thermo-hydraulic behavior of the loop in order to use it for real-time state feedback control, in future applications. First, the one-dimensional two-phase flow model describing the liquid-gas mixture in both mechanical and thermal equilibrium is recalled. The numerical resolution of this detailed model is carried out using a finite volume approach and a Harten-Lax-van Leer Contact Riemann solver. Then, from this detailed model, a new structure of reduced model is determined via the Galerkin projection method. These reduced models, built by the Modal Identification Method, show a very good agreement between the outputs of the detailed model and those computed by the reduced model,
\end{abstract}


during the identification stage. Two test cases, corresponding to different thermal loads at the evaporator, show that the overall levels of density, velocity, mass flow rate, pressure, temperature and internal energy in the loop are satisfactorily reproduced by the reduced model with a global relative error less than $5 \%$. The interest of using such a model lies in the significant gain in CPU time.

Keywords: Stiffened gas equation of state; Two-phase flows model; Compressible flow; Galerkin projection; Low order model; Parameter estimation.

\section{Nomenclature}

a state vector function

A duct cross-section, $\mathrm{m}^{2}$

$B \quad$ coefficient involved in closure laws, $\mathrm{Pa}$

$C_{p} \quad$ specific heat, $\mathrm{J} \cdot \mathrm{kg}^{-1} \cdot \mathrm{K}^{-1}$

$d \quad$ internal diameter, $\mathrm{m}$

$e \quad$ specific internal energy, $\mathrm{J}_{\mathrm{kg}} \mathrm{kg}^{-1}$

$g \quad$ acceleration of gravity, $\mathrm{m} \cdot \mathrm{s}^{-2}$

$h \quad$ specific enthalpy, $\mathrm{J}_{\mathrm{kg}} \mathrm{kg}^{-1}$

$h_{\text {cond }} \quad$ condenser heat exchange coefficient, $\mathrm{W} \cdot \mathrm{m}^{-2} \cdot \mathrm{K}^{-1}$

$H \quad$ output matrix

$\mathcal{J}_{i d}^{(m)} \quad$ quadratic functional to be minimized for order $m$ model identification

$L \quad$ length of the loop, $\mathrm{m}$

$m \quad$ ROM order i.e. size of vector $a$ 


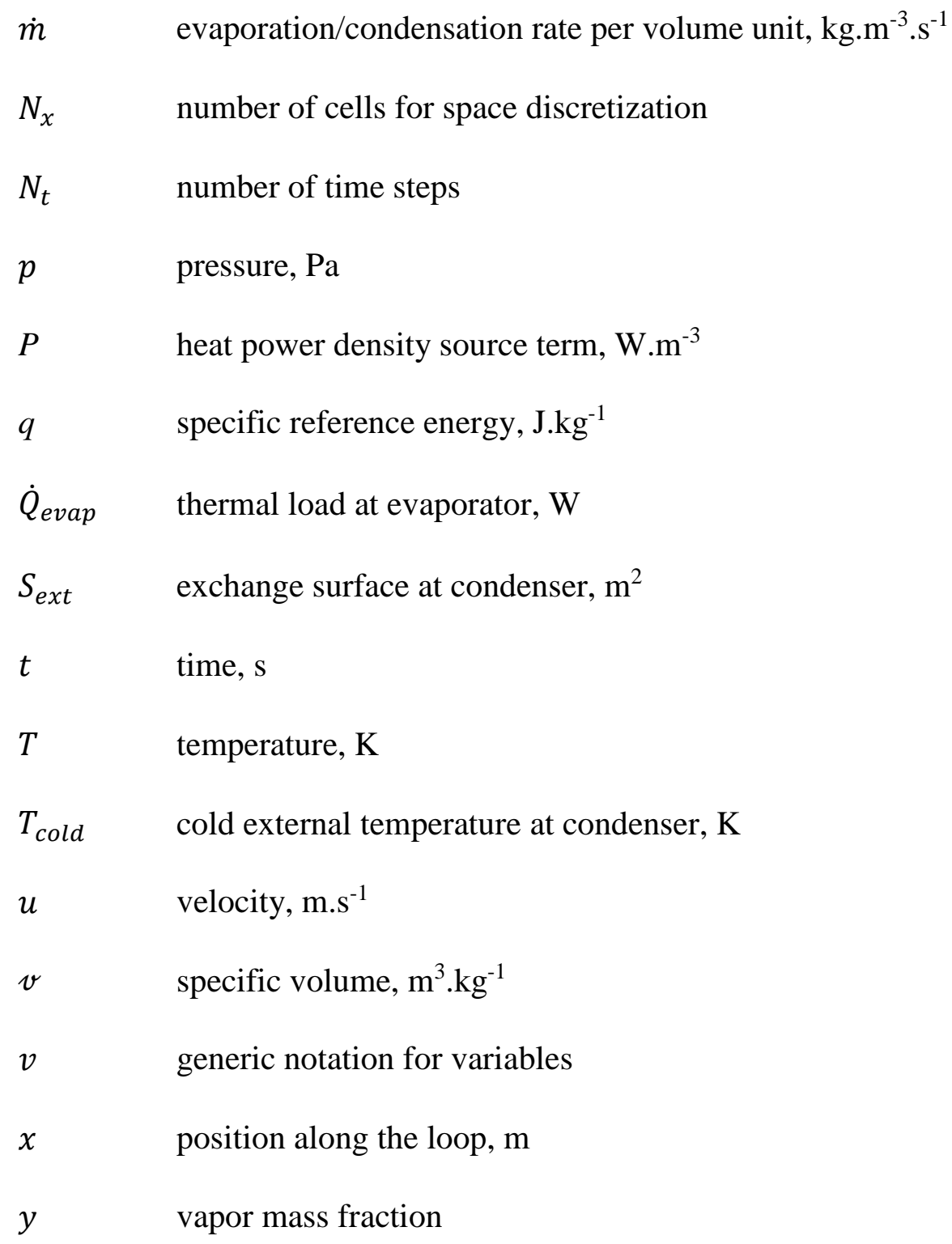




$\begin{array}{ll}\phi_{k}^{(v)} & k^{\text {th }} \text { space function associated with variable } v \\ \mu & \text { dynamic viscosity, } \mathrm{kg} \cdot \mathrm{m}^{-1} \cdot \mathrm{s}^{-1} \\ \rho & \text { density, } \mathrm{kg} \cdot \mathrm{m}^{-3} \\ \Omega & \text { volume } \mathrm{m}^{3}\end{array}$

\section{Subscripts}

$0 \quad$ initial steady state

eq thermodynamic equilibrium

$g \quad$ gas

id identification

$l \quad$ liquid

neq not in thermodynamic equilibrium

Superscripts

$T \quad$ transposition sign

(v) related to variable $v$

Abbreviations

2PLT Two-Phase Loop Thermosyphon

DM Detailed Model

MIM Modal Identification Method

ROM Reduced Order Model

SG Stiffened Gas 


\section{Introduction}

Heat dissipation due to power electronics increases continuously for years, reaching now 300 W.cm ${ }^{-2}$ and beyond, due to electronic components miniaturization. Highly efficient cooling systems are then required. As the classical cooling systems are not powerful enough, an interesting solution consists in using two-phase heat transfer devices, since the cooling capabilities are much larger with latent heat than with sensible heat. Among them, a two-phase loop thermosyphon (2PLT), for which the fluid circulation is generated by buoyancy forces, is a passive cooling system without pump. The working fluid boils at the evaporator due to heat input. The vapor then moves to the condenser where heat is extracted from the system and the vapor changes to liquid. These two-phase closed thermosyphons have been studied in many applications such as, solar water heaters [1] [2] [3] [4], telecommunication equipments [5], avionics systems [6], nuclear power plants [7] [8] [9], electronics industry [10].

Many studies have been carried out on the modeling of such a system in steady and transient states. For instance, Vincent and Kok use 1D control volume approach for the transient behavior of a two-phase co-current thermosyphon [11]. Different models are used for evaporator, condenser, liquid line and vapor line. This means that for computation, each part of loop has its own model. They are based either on hydraulic flow model or compressible flow model. More recently, Bieliński and Mikielewicz present a generalized 1D two-phase separate flow model of the thermosyphon loop [12]. They use incompressible flow model with Boussinesq approximation and empirical correlation in different parts of the loop. This model considers thermal equilibrium at any point of loop. Qu uses two different models for evaporator and condenser [13]. An integral balance of 
bubble flow model is used at the evaporator and the liquid film condensation of vaporliquid concurrent flow model at condenser.

In this paper, a 1D model (Euler equations) is used to describe the transient operation of a simplified loop thermosyphon (constant cross-section, adiabatic liquid and vapor lines, single evaporator) [14]. In each part of the loop, the same model is used in the computation. In other words, the model describes thermodynamic equilibrium (saturation or mixture), but also thermodynamic non-equilibrium (pure liquid or pure vapor) phase and so can compute phase transitions (liquid-mixture-vapor). This simplified model retains the main features of a real 2PLT: gravity-driven two-phase compressible flow, unsteady behavior, thermodynamic equilibrium and non equilibrium. Despite its simplifying assumptions, this model requires large CPU time consumption, even in 1D, especially because of the Courant-Friedrichs-Lewy (CFL) condition. It is hence not usable for real-time applications. In the present paper, this model is used as starting point to build a Reduced Order Model (ROM) of this 2PLT. A ROM is a model involving a small number of degrees of freedom, which reproduces the behavior of an actual system or a reference Detailed Model (DM) with a large number of degrees of freedom, whatever the time-varying boundary conditions and/or source terms.

The goal of this work is to develop a nonlinear ROM able to mimic the thermo-hydraulic behavior of the loop in order to use it for real-time state feedback control, in future applications. To the author's knowledge, this work constitutes the first attempt to develop a ROM of a 2PLT. 
Among model reduction methods for nonlinear problems, let us first cite the Proper Orthogonal Decomposition (POD), also known as Karhunen-Loève decomposition, coupled to a Galerkin projection. As a result of performing POD on discrete space-time data, a set of space functions and time-varying coefficients are obtained, that allow compact approximation of original data. After truncation or selection of modes, a reduced set of space functions is retained for a Galerkin projection of Partial Differential Equations (PDE) onto these space functions, which yields a so-called POD-Galerkin ROM. Although this approach has been widely used in the last decades, especially in fluid mechanics, either for buoyancy-driven flows [15] [16], two-phase flows [17] and compressible flows [18], it is known to often lead to unstable ROMs, even in the case of linear PDE, thus requiring a posteriori stabilization [19].

Another approach is the Reduced Basis (RB) method [20] which aims at building ROMs able to compute solutions of parametrized PDE for given value(s) of parameter(s). It is a two-step approach. In the offline step a reduced basis is built, formed by solutions of a reference Finite Element model at optimally selected points in the parameter space via a Greedy algorithm. The online step consists in solving the ROM which is obtained by a Galerkin projection of PDE onto the reduced basis. Although the RB method can be applied to transient flow problems, directly or by coupling it to POD as done for instance in [21] for natural convection in cavity, it appears to be adapted to the construction of parametric ROMs for parameters which are not time-dependent rather than for timevarying external or internal loads. It should be mentioned that the RB method provides 
errors bounds but in return requires a reference Finite Element model on which it relies [20].

The goal oriented model-constrained approach developed by Bui-Thanh et al. addresses some POD-related issues by computing the basis of space functions via an optimization problem: minimizing the output error between reference model and ROM solutions, subject to satisfying the ROM equations. It is assumed that each basis vector can be represented as a linear combination of snapshots. The approach has been applied in [22] to a compressible flow around a rotor blade, showing that for a small number of approximation functions ( $\leq 10)$, stable ROMs have been obtained whereas POD-Galerkin ROMs were unstable.

The Proper Generalized Decomposition (PGD) does not rely on a reference model and does not make use of simulated or measured data. PGD uses approximations for the variables under separate form, which are sums of products of functions of space coordinates, time and possibly additional parameters. The PGD approximations are computed by successive enrichment. PGD appears to provide a discrete solution, not only in physical space but also in time and parameters space, rather than a continuous ROM in time and parameters. As an example, transient Rayleigh-Bénard flows have been studied in [23].

In the present paper, the Modal Identification Method (MIM) is going to be used for building ROMs for a 2PLT. MIM aims at building ROMs connecting the system inputs (time-varying boundary conditions or sources and/or parameters) to outputs of interest 
(either complete fields of variables, like temperature, velocity, etc., or their value in chosen particular locations). Once the ROM equations are derived from PDE, the ROM's constitutive parameters are identified from input-output data characteristic of the system behavior, through the minimization of an output error. Just as POD, MIM uses either experimental data recorded on the actual system or, as in the present work, simulated data coming from a reference DM.

The MIM has been developed for more than twenty-five years, first for linear problems, and then for several different nonlinear problems. Among them, MIM has been compared to the POD-Galerkin approach on a 3D transient heat diffusion problem with thermal conductivity depending on temperature [24]. A transient inverse heat conduction problem with radiative and convective boundary conditions has been successfully solved with ROMs identified by MIM from experimental data in [25]. MIM has also been applied to non-isothermal flows. In [26], different ROMs have been built for computing a 2D incompressible steady laminar flow over a backward-facing step and associated unsteady forced heat convection. A 2D laminar mixed convection flow around a heated circular cylinder has also been studied in [27]. More recently, ROMs built by MIM from in-situ measurements have been used for efficient handling of real time state feedback control of Multi-Input-Multi-Output thermal systems. Regulation and tracking problems on a ventilated plate, using a mobile heat source as actuator and via a Linear Quadratic Gaussian controller, have been investigated in [28]. Thermal regulation within $0.01^{\circ} \mathrm{C}$ of an ultra-high precision metrology system has also been performed by model predictive control in [29]. 
The paper is structured as follows. The two-phase loop thermosyphon is briefly described in section 2 and the associated flow model is presented in section 3 . The development of the ROM structure and the main features of the MIM are described in section 4. Both identification and validation of ROMs are carried out in section 5.

\section{Description of the two-phase loop thermosyphon}

The Two-Phase Loop Thermosyphon (2PTL) is a passive thermal system, as depicted in Figure 1. It is composed of an evaporator, a vapor line, a condenser and a liquid line. The working fluid (methanol in our case) is heated (heat flux $\dot{Q}_{\text {evap }}$ ) and vaporized in the evaporator $(B-C)$. The vapor then flows through the vapor line (C-E) toward the condenser which is a low temperature heat exchanger (cold external temperature $T_{\text {cold }}$ and global heat transfer coefficient $\left.h_{\text {cond }}\right)$. The vapor is cooled in the condenser and turns into liquid which falls under gravity effect in the vertical line (F-A). This liquid is then driven toward the evaporator by the horizontal line $(A-B)$ where it is heated again. Such a specific configuration, with a heat sink located higher than the heat source, enables a natural circulation inside the loop. The total length of the loop is equal to $1.5 \mathrm{~m}$. The tube cross-section $A$ is assumed constant along the loop with an internal diameter $d$ equal to $0.007 \mathrm{~m}$. 


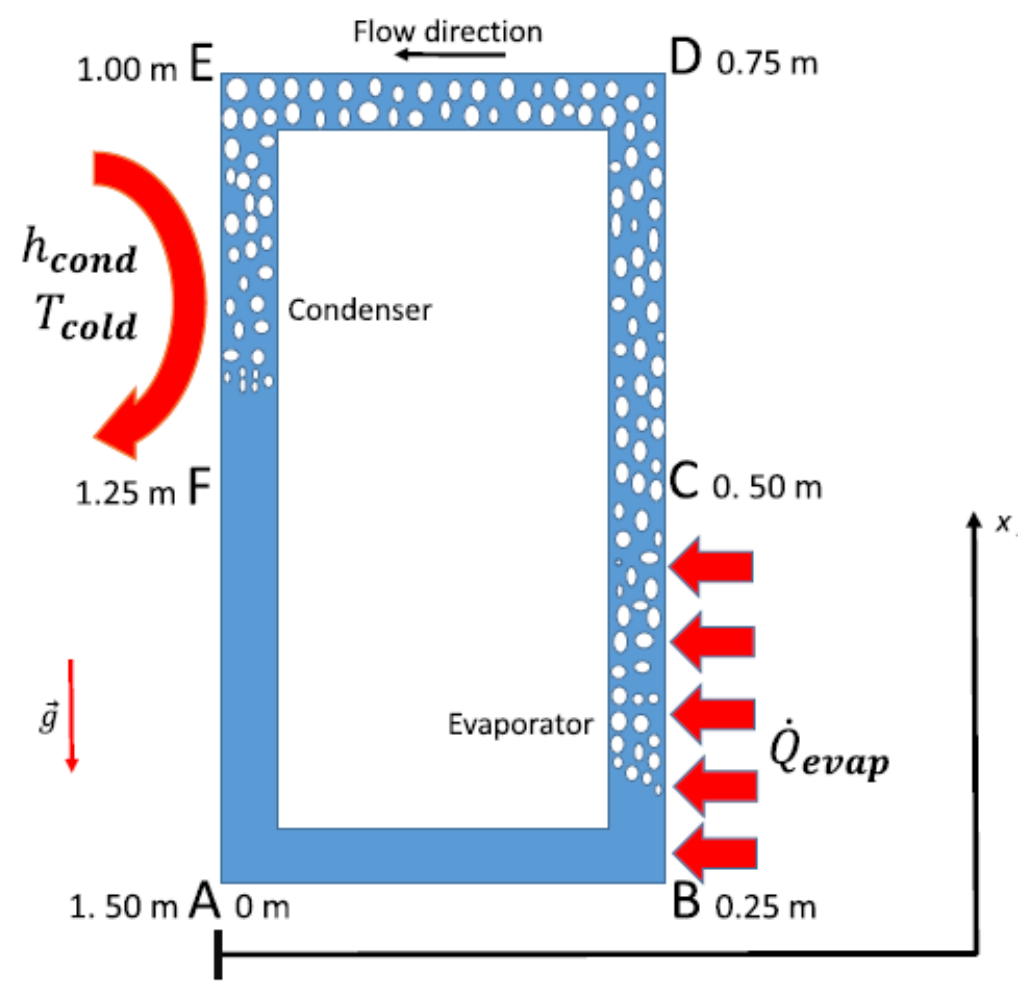

Figure 1 - The two-phase loop thermosyphon and its boundary conditions.

\section{Flow model of the loop}

\subsection{Assumptions}

As the objective of the paper is not to study accurately the behavior of the loop but to propose a reduced model structure able to take into account the phase change in the thermal systems, some assumptions have been made in order to simplify the flow model:

- The fluid mixture is compressible as both heating and phase change make the mixture density in the loop change with space and time;

- The two-phase flow is considered as a homogeneous mixture in both mechanical and thermal equilibrium: same velocity, pressure and temperature for both phases at a given location; 
- Except at the evaporator and the condenser, the duct is supposed adiabatic;

- The heat exchange coefficient $h_{\text {cond }}$ and the exchange surface $S_{\text {ext }}$ at the condenser are constant;

- Heat diffusion inside the mixture is neglected;

- The Darcy-Weisbach law is used to model the friction effects. As the regime is supposed laminar, the friction factor is equal to $64 / R e$ where $R e$ denotes the Reynolds number.

- The thermal inertia of the duct wall depth of the loop is not taken into account.

\subsection{Flow model}

The equations of the one-dimensional, one or two-phase compressible flow in transient state are given by, $x$ being the rectangular coordinate along the loop and $t$ the time:

$$
\begin{gathered}
\frac{\partial \rho}{\partial t}+\frac{\partial(\rho u)}{\partial x}=0 \\
\frac{\partial(\rho u)}{\partial t}+\frac{\partial\left(\rho u^{2}+p\right)}{\partial x}=-\frac{64 \mu}{2 d^{2}} u+\epsilon \rho g \\
\frac{\partial(\rho e)}{\partial t}+\frac{\partial(\rho e u)}{\partial x}=-p \frac{\partial u}{\partial x}+\frac{64 \mu}{2 d^{2}} u^{2}+P \\
\frac{\partial(\rho y)}{\partial t}+\frac{\partial(\rho y u)}{\partial x}=\dot{m}
\end{gathered}
$$

where $\rho, u, p, e$, and y are the mixture density, velocity, pressure, specific internal energy and vapor mass fraction respectively. $\dot{m}$ is the evaporation/condensation rate per volume unit. The dynamic viscosity $\mu$ was considered in [14] as a function of mass fraction and temperature. However, in this first attempt to build a ROM for the 2PLT, in 
order to simplify the ROM formulation, $\mu$ is supposed to be the same in both liquid and vapor phases. It is assumed to be constant and equal to the liquid's dynamic viscosity. The gravity effects are modeled through the $\epsilon \rho g$ term where $\epsilon$ is as follows:

$$
\epsilon=\left\{\begin{array}{l}
-1 \text { if } x \in[B D] \\
0 \text { if } x \in[A B] \cup[D E] \\
1 \text { if } x \in[E A]
\end{array}\right.
$$

The thermal boundary conditions at the evaporator and the condenser are modeled by a source term $P$ in the internal energy equation (3) defined by:

$$
P=\left\{\begin{array}{l}
\dot{Q}_{\text {evap }} / \Omega_{\text {evap }} \text { if } x \in[B C] \\
0 \text { if } x \in[C E] \cup[F B] \\
h_{\text {cond }} S_{\text {ext }}\left(T_{\text {cold }}-T\right) / \Omega_{\text {cond }} \text { if } x \in[E F]
\end{array}\right.
$$

where $\dot{Q}_{\text {evap }}$ is the thermal load at the evaporator whose volume is $\Omega_{\text {evap }} . h_{\text {cond }}$, $\Omega_{\text {cond }}, S_{\text {ext }}$ and $T_{\text {cold }}$ are the heat exchange coefficient, the condenser volume, the exchange surface and the cold external temperature at the condenser, respectively. $T$ is the fluid mixture temperature.

This two-phase flow model requires the equation of state for each pure phase of the methanol and for the liquid-vapor mixture. As the finite volume method coupled with the approximate Riemann solver called Harten - Lax - van Leer - Contact (HLLC) numerical scheme is used to solve the problem, the sound speed computation is needed. In order to avoid imaginary values of this sound speed in the mixture zone, a "Stiffened Gas" (SG) equation of state is used [30].

At any location along the loop, the fluid is either in or out of thermodynamic equilibrium. The thermodynamic equilibrium condition corresponds to the equality between Gibbs 
free energies: $G_{g}=G_{l}$ where $l$ and $g$ denote liquid phase and vapor phase respectively. For each phase $k=l$ or $g$, Gibbs free energy $G_{k}$ for a SG equation of state is given by:

$$
G_{k}(p, T)=\left(\gamma_{k} C_{v, k}-q_{k}^{\prime}\right) T-C_{v, k} T \ln \left(\frac{T^{\gamma_{k}}}{\left(p+p_{\infty, k}\right)^{\gamma_{k}-1}}\right)+q_{k}, \quad k=l \text { or } g
$$

Coefficients $C_{v, k}, \gamma_{k}$ and $p_{\infty, k}$ are constant quantities determined in such a way that specific enthalpy $h_{k}$, specific volume $v_{k}=1 / \rho_{k}$ and latent heat vaporization $h_{l v}=$ $h_{g}^{\text {sat }}-h_{l}^{\text {sat }}$ fit with the experimental values provided by Goodwin in the $280 \mathrm{~K}-370 \mathrm{~K}$ temperature range [31]. $C_{v, k}, \gamma_{k}$ and $p_{\infty, k}$ are determined using four points $\left(T_{i}, p_{\text {sat }}\left(T_{i}\right)\right), i \in\{0, \ldots, 3\}$ on the saturation curve and their values are given in [14], as well as the SG formulae for the computation of $h_{k}, v_{k}$ and $q_{k}$.

It has been shown in [30] that the condition $G_{g}=G_{l}$ leads to a relation $f(p, T)$ depending on the difference $q_{g}^{\prime}-q_{l}^{\prime} . q_{l}^{\prime}$ is thus set equal to zero and $q_{g}^{\prime}$ is determined so that the relation $f(p, T)$ fits with the experimental saturation curve provided by Goodwin in [31].

The thermodynamic equilibrium condition is thus replaced by the following conditions:

$$
0<y<1 \text { and } T=T_{\text {sat }}(p)
$$

It means that the thermodynamic equilibrium corresponds to a two-phase fluid in saturation state whereas the out of thermodynamic equilibrium situation corresponds to a one-phase or an unsaturated fluid.

The closure laws of eqs. (1)-(4) write:

- Out of thermodynamic equilibrium: 


$$
\left\{\begin{array}{c}
p=\frac{1}{2}\left[B_{g}+B_{l}-\left(p_{\infty, g}+p_{\infty, l}\right)\right]+\left(\frac{1}{4}\left[B_{l}-B_{g}-\left(p_{\infty, l}-p_{\infty, g}\right)\right]^{2}+B_{g} B_{l}\right)^{1 / 2} \\
\frac{1}{\rho T}=\frac{y_{g}\left(\gamma_{g}-1\right) C_{v, g}}{p+p_{\infty, g}}+\frac{y_{l}\left(\gamma_{l}-1\right) C_{v, l}}{p+p_{\infty, l}}
\end{array}\right.
$$

The vapor mass fraction is $y_{g}=1-y_{l}=y$ and the formula for computing $B_{k}, k=l$ or $g$, is given in [14].

- In thermodynamic equilibrium:

$$
\left\{\begin{array}{c}
v=y_{g}^{*} v_{g}\left(p^{*}\right)+y_{l}^{*} v_{l}\left(p^{*}\right) \\
e=y_{g}^{*} e_{g}\left(p^{*}\right)+y_{l}^{*} e_{l}\left(p^{*}\right) \\
T^{*}=T_{\text {sat }}\left(p^{*}\right)
\end{array}\right.
$$

After eliminating $y_{g}^{*}=1-y_{l}^{*}$ by combining the first two equations of (10) and using the definition of specific enthalpy $h$, we get:

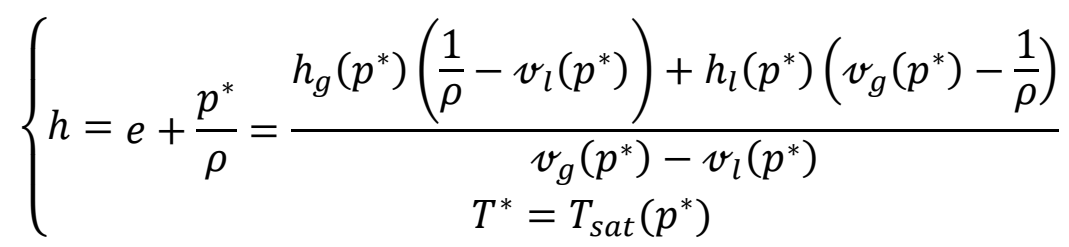

In each case (in or out of thermodynamic equilibrium), the first equation is used to compute pressure $p$ and then the second one is used to obtain temperature $T$.

The flow model is solved with a Godunov's first order method and a Harten - Lax - van Leer - Contact (HLLC) Riemann solver [32]. The flow solver is described in detail in [14]. The schematic algorithm of the Detailed Model is given in Figure 2. 


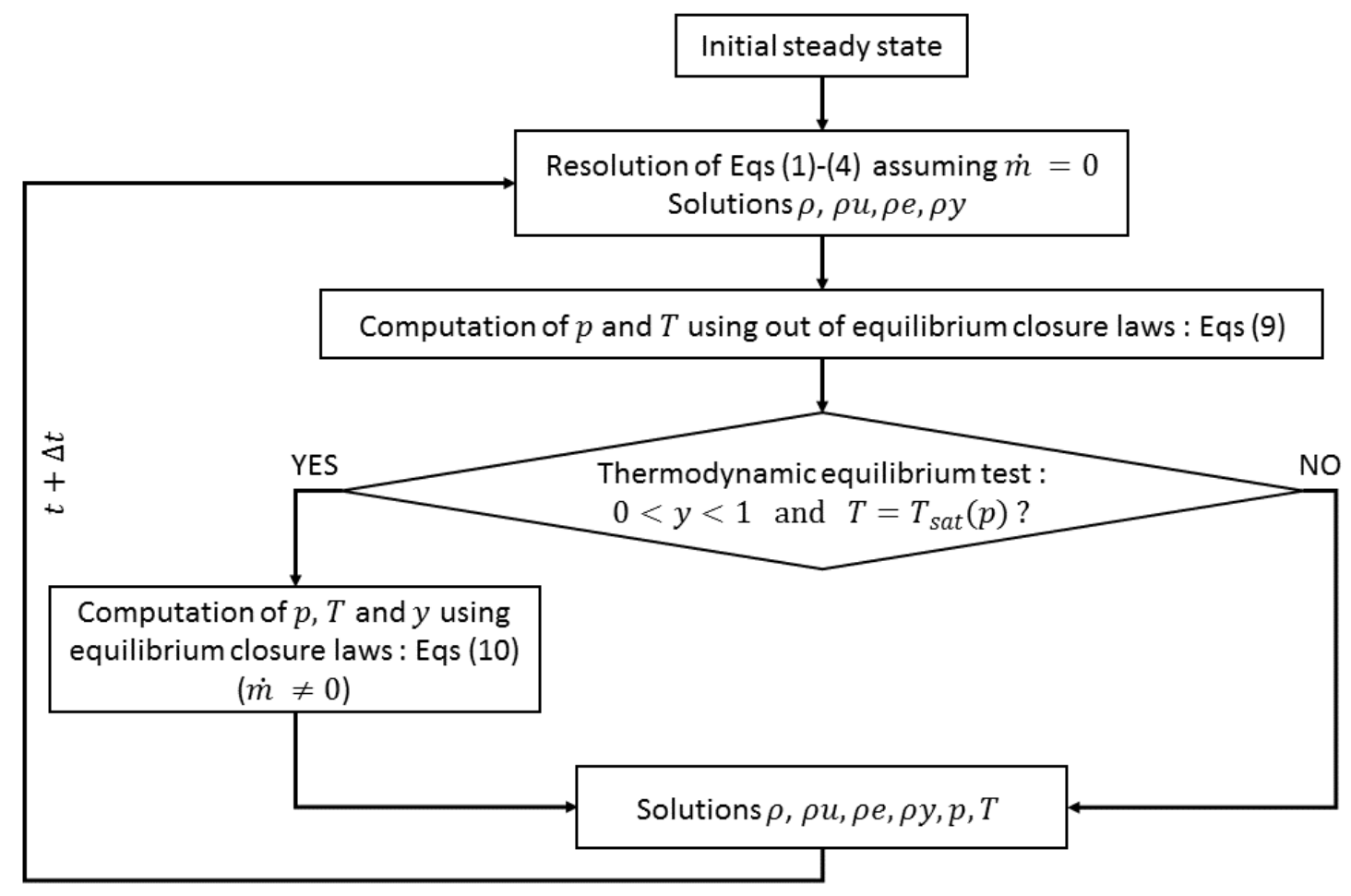

Figure 2: schematic algorithm of Detailed Model

\section{Reduced order model by modal identification method}

Our objective is to build a Reduced Order Model able to quickly compute fields of variables close to those coming from the reference model, whatever the heat power applied to the evaporator.

\subsection{Modal Identification Method Overview}

The Modal Identification Method (MIM) consists in three main steps:

1) Definition of the ROM equations structure, starting from local equations (see section 4.2). This is the core of the paper; 
2) Generation of some input-output data characteristic of the system dynamics. Those data may come from in-situ measurements or, like in the present paper, from numerical simulations (see section 4.3);

3) Identification of the ROM fixed constitutive parameters through the minimization of a functional based on the quadratic residuals between the previously generated output data of the system on the one hand and the outputs of the ROM on the other hand, for the same input data, here a specific time-varying heat power applied to the evaporator (see section 4.4).

\subsection{Reduced model formulation}

Two ROMs are going to be presented in the following. The first one may be called "full" ROM, but features some important issues. The second one is a simpler ROM which requires shortcuts, but allows to overcome the drawbacks of the first ROM.

\subsubsection{A first approach: "full" ROM}

This first ROM formulation:

- is derived from the whole set of equations: conservation equations (1)-(4) along with equations (5) and (6) and closure laws (9) and (11);

- is based on primitive variables $\rho, u, e, y, p, T$;

- uses approximations of the variables fields with a specific set of dynamics for each variable $v$ : 


$$
v(x, t)=\sum_{i=1}^{m} a_{i}^{(v)}(t) \phi_{i}^{(v)}(x) \quad \forall v \in\{\rho, u, e, y, p, T\}
$$

where for each variable $v$, space functions $\phi_{i}^{(v)}(x), i=1, \ldots, m$, are a truncation of a basis of the Hilbert space formed by the $\mathcal{L}^{2}(\Omega=[0 ; L])$ space of square integrable functions on $\Omega=[0 ; L]$ equipped with the usual inner product $(., .)_{\Omega}$ :

$$
(f, g)_{\Omega}=\int_{0}^{L} f g d x
$$

where functions $f$ and $g$ are defined on $\Omega$.

Of course, our goal is to find a ROM, thus corresponding to a small number $m$ of functions used in the decomposition of variables fields. It should be noticed that different numbers of functions could have been considered for the different variables. For the sake of simplicity, the same number of functions $m$ is here used for all variables. $m$ is called the order of the ROM.

Let us call:

- $R^{(v)}(x, t), v \in\{\rho, u, e, y\}$, the residuals of conservation equations (1)-(4);

- $R_{\text {neq }}^{(v)}(x, t), v \in\{p, T\}$ the residuals of eqs.(9) in developed form;

- $R_{e q}^{(v)}(x, t), v \in\{p, T\}$ the residuals of eqs.(11) in developed form.

The Galerkin projection consists in forcing each residual, written with approximations of variables defined by equation (12), to be orthogonal to each space function of the corresponding set according to the inner product (13), so that the projection of the residual onto the subspace of $\mathcal{L}^{2}(\Omega)$ generated by the set of space functions would be null. The following equations are thus written. 
For conservation equations (1)-(4):

$$
\begin{gathered}
\left(R^{(v)}(x, t), \phi_{k}^{(v)}(x)\right)_{\Omega}=\int_{0}^{L} R^{(v)}(x, t) \phi_{k}^{(v)}(x) d x=0 \\
\forall k=1, \ldots, m, \quad \forall v \in\{\rho, u, e, y\}
\end{gathered}
$$

For closure laws (9) (out of thermodynamic equilibrium):

$$
\begin{gathered}
\left(R_{\text {neq }}^{(v)}(x, t), \phi_{k}^{(v)}(x)\right)_{\Omega}=\int_{0}^{L} R_{\text {neq }}^{(v)}(x, t) \phi_{k}^{(v)}(x) d x=0 \\
\forall k=1, \ldots, m, \quad \forall v \in\{p, T\}
\end{gathered}
$$

For closure laws (11) (thermodynamic equilibrium):

$$
\begin{gathered}
\left(R_{e q}^{(v)}(x, t), \phi_{k}^{(v)}(x)\right)_{\Omega}=\int_{0}^{L} R_{e q}^{(v)}(x, t) \phi_{k}^{(v)}(x) d x=0 \\
\forall k=1, \ldots, m, \quad \forall v \in\{p, T\}
\end{gathered}
$$

Since the development of Galerkin projections is too long to be detailed and the resulting sets of equations are too big to be written in the present paper, the general form is given in the following in order to highlight the most crucial points.

Let us define the vector-valued function $\mathcal{A}(t) \in \mathbb{R}^{6 m}$ :

$$
\mathcal{A}(t)=\left(\begin{array}{llllll}
a^{(\rho)}(t) & a^{(u)}(t) & a^{(e)}(t) & a^{(y)}(t) & a^{(p)}(t) & \left.a^{(T)}(t)\right)^{T}
\end{array}\right.
$$

The Galerkin projections (14) of the four local PDE lead to a set of four systems, each being composed of $m$ equations. Those systems can be gathered as follows:

$$
\frac{d}{d t}\left(F_{0}(\mathcal{A}(t), \theta)\right)=F_{1}\left(\mathcal{A}(t), \theta, \dot{Q}_{\text {evap }}(t)\right)
$$

where $F_{0} \in \mathbb{R}^{4 m}$ and $F_{1} \in \mathbb{R}^{4 m}$ are vector-valued functions that are not detailed here. 
The Galerkin projections (15) and (16) of the two sets of closure laws (for thermodynamic non-equilibrium and equilibrium situations respectively), yield two sets of two systems, each being composed of $m$ equations:

$$
\begin{gathered}
F_{\text {neq }}(\mathcal{A}(t), \theta)=0 \\
F_{\text {eq }}(\mathcal{A}(t), \theta)=0
\end{gathered}
$$

$\theta$ is a vector gathering the fixed constitutive parameters of the dynamical system of equations in the ROM.

The ROM stemming from this first approach is thus composed of:

- equations (17), (18) and (19) allowing the computation of the global low order state vector $\mathcal{A}(t) \in \mathbb{R}^{6 m}$ with respect to $\dot{Q}_{\text {evap }}(t)$;

- equation (12) allowing the reconstruction of fields of variables $\rho, u, e, y, p, T$ once $\mathcal{A}(t)$ is computed.

\subsubsection{Issues connected to $\mathrm{ROM} \mathrm{n}^{\circ} 1$}

Four issues arise from this first ROM formulation:

1. The first system of $m$ equations in (17) comes from the Galerkin projection of mass conservation equation (1). It writes:

$$
\begin{gathered}
\int_{0}^{L} R^{(\rho)}(x, t) \phi_{k}^{(\rho)}(x) d x=\sum_{i=1}^{m} M_{k i} \frac{d a_{i}^{(\rho)}(t)}{d t}+\sum_{i=1}^{m} \sum_{j=1}^{m} Q_{k i j} a_{i}^{(\rho)}(t) a_{j}^{(u)}(t)=0 \\
\forall k=1, \ldots, m
\end{gathered}
$$

where $M \in \mathbb{R}^{m \times m}$ and $Q \in \mathbb{R}^{m \times m \times m}$ are two tensors depending on space functions. 
In steady state, equation (20) reduces to:

$$
\sum_{i=1}^{m} \sum_{j=1}^{m} Q_{k i j} a_{i}^{(\rho)} a_{j}^{(u)}=0 \quad \forall k=1, \ldots, m
$$

This equation has to be verified for any vectors $a^{(\rho)}$ and $a^{(u)}$ corresponding to a steady state associated to a constant $\dot{Q}_{\text {evap }}$ thermal load. All components of tensor $Q$ have thus to be zero. As a consequence, equation (20) would write:

$$
\sum_{i=1}^{m} M_{k i} \frac{d a_{i}^{(\rho)}(t)}{d t}=0 \quad \forall k=1, \ldots, m
$$

Then either tensor $M$ is null and the Galerkin projection of mass conservation equation no longer exists - we would then have 5 systems of $m$ equations for $6 m$ unknowns - or the $a_{i}^{(\rho)}$ never vary with time and hence does density $\rho$. None of these situations is acceptable.

2. In order to close the system (17), one has to use either equation (19) or equation (18), depending on whether the fluid is in thermodynamic equilibrium or not. Whereas for the DM, the choice between equations (9) and equations (11) is made according to the local test (8) at each instant (the fluid may thus be in thermodynamic equilibrium in some parts of the loop and out of equilibrium in other parts at a given instant), this is not possible with the ROM. Due to the decoupling of time and space in the approximations of variables (12), equations (18) and (19) are only time-dependent, meaning that at each instant, the fluid all along the loop is either in thermodynamic equilibrium or out of equilibrium. 
3. Transport terms in PDE (2), (3) and (4), which are of third degree when using $\rho, u, e, y$ as variables, lead to terms involving order 4 tensors (of size $m^{4}$ ) in the right hand side of the ROM equation (17). Once written in developed form to avoid fractions, closure laws (9) and (11) show terms of third and fourth degree which respectively bring order 4 tensors (of size $\mathrm{m}^{4}$ ) and order 5 tensors (of size $\left.m^{5}\right)$ in the ROM equations (18) and (19). In the end, the number $n_{\theta}\left(R O M n^{\circ} 1\right)$ of parameters in vector $\theta$ appearing in equations (17), (18) and (19) is:

$$
n_{\theta}\left(R O M n^{\circ} 1\right)=5 m^{5}+15 m^{4}+16 m^{3}+13 m^{2}+7 m
$$

The number of parameters to be identified for the ROM construction thus strongly increases with respect to the ROM order $m$.

4. Due to the use of $\rho, u, e, y$ as variables, inertia terms in PDE (2), (3) and (4) lead respectively to terms of the form $\frac{d\left(a_{i}^{(\rho)}(t) a_{j}^{(u)}(t)\right)}{d t}, \frac{d\left(a_{i}^{(\rho)}(t) a_{j}^{(e)}(t)\right)}{d t}$ and $\frac{d\left(a_{i}^{(\rho)}(t) a_{j}^{(y)}(t)\right)}{d t}$ in the left hand side of the ROM equation (17). Such time derivatives are not easy to handle.

Based on the aforementioned issues, new assumptions are going to be made in the following in order to work on a simplified ROM formulation.

\subsubsection{Solutions proposed to address issues of ROM ${ }^{\circ} 1$}

The solutions proposed to overcome the aforementioned issues are given hereinafter:

1. In order to avoid the issue $n^{\circ} 1$ of section 4.2.2, mass conservation equation (1) can be combined with at least another equation when performing the Galerkin 
projection, thus corresponding to a partial or complete vector Galerkin projection.

2. The handling of closure laws associated with the Stiffened Gas equation of state is quite a complex problem, which induces difficulties in the ROM for both the choice of the closure equations (issue $n^{\circ} 2$ of section 4.2.2) and the number of parameters in the model because of tensors of order 4 and 5 (issue $n^{\circ} 3$ of section 4.2.2). As a consequence, we are going to ignore closure laws (9) and (11) for deriving the ROM formulation. Such shortcut may seem quite severe but will prove to be efficient. One should note that the ROM will still be built from numerical data composed of fields of variables computed with the DM for which closure laws (9) and (11) are used. The choice of not taking into account these closure laws for defining the ROM equations has two immediate consequences:

- Firstly, equations for the computation of $a^{(p)}(t)$ and $a^{(T)}(t)$ respectively associated with pressure and temperature will be missing in the ROM. The reconstruction of $p$ and $T$ will hence require to replace sets of dynamics $a^{(p)}(t)$ and $a^{(T)}(t)$ by sets of dynamics associated with other variables. The corresponding space-time decompositions will be introduced in the Galerkin projections of equations (2) and (3);

- Secondly, as vapor mass fraction $y$ only appears in PDE (4) and closure laws (9) and (11), the variable $y$ will not be considered and equation (4) will not be needed anymore. One should note that $y$ could still be obtained via the knowledge of density $\rho$, if desired. 
3. Conservative variables $\rho, \rho u, \rho e$ will be used instead of primitive variables $\rho, u, e$. This will allow to decrease from third degree to second degree the transport terms in PDE (2) and (3) and hence to move from order 4 to order 3 tensors. This will enable to decrease the number of parameters in the ROM (issue $\mathrm{n}^{\circ} 3$ of section 4.2.2). In addition, inertia terms in PDE will move from second degree to first degree, thus resulting in first degree time derivatives in the ROM (issue $\mathrm{n}^{\circ} 4$ of section 4.2.2). However, velocity $u$ will still be present in equations (2) and (3). Variables $\rho, \rho u$ and $u$ are not independent and are linked by the simple relation $\rho u=\rho \times u$.

Finally, equations that will be considered for deriving the new ROM formulation are conservation equations (1)-(3) along with eqs.(5) and (6) and equation $\rho u=\rho \times u$.

\subsubsection{Equations for deviations with respect to initial steady state}

Before advancing to $\operatorname{ROM} \mathrm{n}^{\circ} 2$, equations for deviations with respect to initial steady state are given. This initial steady state is considered to be the same for all our studied cases and corresponds to a thermosyphon loop with the bottom half-filled with liquid methanol $\left(\rho_{0}=853 \mathrm{~kg} \cdot \mathrm{m}^{-3}\right.$ and $\left.y_{0}=0\right)$ and the upper half-filled with vapor methanol $\left(\rho_{0}=10^{-1} \mathrm{~kg} \cdot \mathrm{m}^{-3}\right.$ and $\left.y_{0}=1\right)$, no circulation (fluid at rest i.e. $\left.u_{0}=(\rho u)_{0}=0\right)$, no heat power applied to the evaporator $\left(\dot{Q}_{\text {evap }}=0\right)$ and fluid at the external temperature $\left(T_{0}=T_{\text {cold }}\right)$. The only remaining equation is the momentum conservation equation (2) which writes: 


$$
\frac{\partial p_{0}}{\partial x}=\epsilon \rho_{0} g
$$

The internal energy $e_{0}$ is obtained by the Stiffened Gas model from pressure $p_{0}$, temperature $T_{0}$ and vapor mass fraction $y_{0}$.

Let us define the deviations of the variables $\rho, \rho u, \rho e, u, p, T$ with respect to the fields in initial steady state:

$$
\delta v(x, t)=v(x, t)-v_{0}(x), \quad v \in\{\rho, \rho u, \rho e, u, p, T\}
$$

Plugging deviations (23) in conservation equations (1)-(3) and equation $\rho u=\rho \times u$ and taking into account equation (22) leads to the following set of equations for the deviations, where $R^{(\delta v)}$ refers to the residual of the equation associated to variable $\delta v$ :

$$
\begin{gathered}
R^{(\delta \rho)}(x, t)=\frac{\partial(\delta \rho)}{\partial t}+\frac{\partial(\delta(\rho u))}{\partial x}=0 \\
R^{(\delta(\rho u))}(x, t)=\frac{\partial(\delta(\rho u))}{\partial t}+\frac{\partial(\delta(\rho u) \delta u)}{\partial x}+\frac{\partial(\delta p)}{\partial x}+\frac{64 \mu}{2 d^{2}} \delta u-\epsilon \delta \rho g=0 \\
R^{(\delta(\rho e))}(x, t)=\frac{\partial(\delta(\rho e))}{\partial t}+\frac{\partial(\delta(\rho e) \delta u)}{\partial x}+\delta p \frac{\partial(\delta u)}{\partial x}-\frac{64 \mu}{2 d^{2}}(\delta u)^{2}-P \\
+\frac{\partial\left(\delta u(\rho e)_{0}\right)}{\partial x}+p_{0} \frac{\partial \delta u}{\partial x}=0 \\
R^{(\delta u)}(x, t)=\delta(\rho u)-\delta \rho \delta u-\rho_{0} \delta u=0
\end{gathered}
$$

where $\epsilon$ is still defined by eq.(5) and heat source term $P$ is still given by eq.(6) where $T_{\text {cold }}=T_{0}$ and thus $T_{\text {cold }}-T=T_{0}-T=-\delta T(x, t)$ according to (23).

\subsubsection{A second approach: simpler $\mathrm{ROM} \mathrm{n}^{\circ} 2$}

The second ROM formulation: 
- is derived from the set of conservation equations (24)-(26) along with eqs.(5) and (6) and from equation (27); According to point $n^{\circ} 2$ of section 4.2.3, closure laws written for the deviations of variables are not taken into account;

- $\quad$ is based on deviations $\delta \rho, \delta(\rho u), \delta(\rho e), \delta u, \delta p, \delta T$ of conservative variables with respect to the variable fields in initial steady state;

- uses approximations of the deviations fields with a unique set of dynamics for the deviation $\delta v$ of each variable $v$ :

$$
\delta v(x, t)=\sum_{i=1}^{m} a_{i}(t) \phi_{i}^{(\delta v)}(x) \quad \forall v \in\{\rho, \rho u, \rho e, u, p, T\}
$$

It should be noted that, as equations (24) to (27) are used and because the mass conservation equation (24) needs to be combined with at least another equation according to point $n^{\circ} 1$ of section 4.2 .3 , we could have opted for either:

- The combination of equation (24) with one of the three other equations, thus performing three Galerkin projections (one vector and two scalar projections) corresponding to three sets of dynamics for our six variables;

- The combination of equation (24) with two of the three other equations, thus performing two Galerkin projections (one vector and one scalar projection) corresponding to two sets of dynamics for our six variables;

- The combination of equation (24) with the three other equations, thus performing a single vector Galerkin projection corresponding to a single set of dynamics for our six variables; 
The last option has been chosen in order to investigate the quality of the simplest possible nonlinear ROM with the lowest possible number of constitutive parameters. Let us define:

- the vector $\vec{R}(x, t)$ of residuals corresponding to equations (24) to (27):

$$
\vec{R}(x, t)=\left(\begin{array}{c}
R^{(\delta \rho)}(x, t) \\
R^{(\delta(\rho u))}(x, t) \\
R^{(\delta(\rho e))}(x, t) \\
R^{(\delta u)}(x, t)
\end{array}\right)
$$

- vectors $\overrightarrow{\phi_{k}}(x), k=1, \ldots, m$ of space functions associated with deviations $\delta v$, $v \in\{\rho, \rho u, \rho e, u\}:$

$$
\overrightarrow{\phi_{k}}(x)=\left(\begin{array}{c}
\phi_{k}^{(\delta \rho)}(x) \\
\phi_{k}^{(\delta(\rho u))}(x) \\
\phi_{k}^{(\delta(\rho e))}(x) \\
\phi_{k}^{(\delta u)}(x)
\end{array}\right) \quad \forall k=1, \ldots, m
$$

The inner product considered is now:

$$
(\vec{f}, \vec{g})_{\Omega}=\int_{0}^{L} \vec{f} \cdot \vec{g} d x
$$

Which generalizes the inner product (13) to vector functions $\vec{f}$ and $\vec{g}$.

The vector Galerkin projection consists in forcing the residual $\vec{R}(x, t)$ defined by eq.(29) and written with approximations of deviations defined by eq.(28), to be orthogonal to each space vector function $\overrightarrow{\phi_{k}}(x), k=1, \ldots, m$ defined by eq.(30) according to the inner product (31), so that the projection of the residual onto the subspace of $\mathcal{L}^{2}(\Omega)$ generated by the set of space functions would be null. The following equation is thus written: 


$$
\left(\vec{R}(x, t), \overrightarrow{\phi_{k}}(x)\right)_{\Omega}=\int_{0}^{L} \vec{R}(x, t) \cdot \overrightarrow{\phi_{k}}(x) d x=0 \quad \forall k=1, \ldots, m
$$

The four different terms of the projection (32) are given in Appendix A and the expressions of the involved tensors are shown in Appendix B.

Equation (32) may be written as:

$$
\begin{gathered}
\sum_{i=1}^{m}\left(M_{0}\right)_{k i} \frac{d a_{i}(t)}{d t}+\sum_{i=1}^{m} \sum_{j=1}^{m}\left(Q_{0}\right)_{k i j} a_{i}(t) a_{j}(t)+\sum_{i=1}^{m}\left(L_{0}\right)_{k i} a_{i}(t)+\left(V_{0}\right)_{k} \dot{Q}_{e v a p}(t)=0 \\
\forall k=1, \ldots, m
\end{gathered}
$$

The proof that matrix $M_{0} \in \mathbb{R}^{m \times m}$ is invertible is given in Appendix C. Defining tensors $Q \in \mathbb{R}^{m \times m \times m}, L \in \mathbb{R}^{m \times m}$ and $V \in \mathbb{R}^{m}$ as $Q=\left(M_{0}\right)^{-1} Q_{0}, L=\left(M_{0}\right)^{-1} L_{0}$ and $V=$ $\left(M_{0}\right)^{-1} V_{0}$ respectively, we get from equation (33):

$$
\frac{d a_{k}(t)}{d t}+\sum_{i=1}^{m} \sum_{j=1}^{m} Q_{k i j} a_{i}(t) a_{j}(t)+\sum_{i=1}^{m} L_{k i} a_{i}(t)+V_{k} \dot{Q}_{\text {evap }}(t)=0 \quad \forall k=1, \ldots, m
$$

Let us call $\theta$ the vector gathering components of tensors $Q, L, V$ in equation (34). The number $n_{\theta}\left(R O M n^{\circ} 2\right)$ of parameters in vector $\theta$ is:

$$
n_{\theta}\left(R O M n^{\circ} 2\right)=m^{3}+m^{2}+m
$$

Equation (28) is now written for all $N_{x}$ points of the detailed model mesh:

$$
\delta v\left(x_{j}, t\right)=\sum_{i=1}^{m} a_{i}(t) \phi_{i}^{(\delta v)}\left(x_{j}\right) \quad \forall j=1, \ldots, N_{x} \quad \forall v \in\{\rho, \rho u, \rho e, u, p, T\}
$$

Let us define matrices $H^{(\delta v)} \in \mathbb{R}^{N_{x} \times m}$ such as:

$$
H_{j i}^{(\delta v)}=\phi_{i}^{(\delta v)}\left(x_{j}\right) \quad \forall j=1, \ldots, N_{x} \quad \forall i=1, \ldots, m \quad \forall v \in\{\rho, \rho u, \rho e, u, p, T\}
$$

Using (37), equation (36) is written as: 


$$
\delta v\left(x_{j}, t\right)=\sum_{i=1}^{m} H_{j i}^{(\delta v)} a_{i}(t) \quad \forall j=1, \ldots, N_{x} \quad \forall v \in\{\rho, \rho u, \rho e, u, p, T\}
$$

For easier reference to matrices $H^{(\delta v)} \in \mathbb{R}^{N_{x} \times m}, v \in\{\rho, \rho u, \rho e, u, p, T\}$, these can be gathered in macro-matrix $H \in \mathbb{R}^{6 N_{x} \times m}$ :

$$
H=\left(H^{(\delta \rho)}, \quad \cdots \quad, H^{(\delta T)}\right)^{T}
$$

The simpler ROM stemming from this second approach is thus composed of:

- equation (34) allowing the computation of the low order state vector $a(t) \in \mathbb{R}^{m}$ whose components are the $a_{i}(t), i=1, \ldots, m$, with respect to $\dot{Q}_{\text {evap }}(t)$;

- equation (38) allowing the reconstruction of discrete fields of variables $\delta \rho, \delta(\rho u), \delta(\rho e), \delta u, \delta p, \delta T$ at each instant, once $a(t)$ is computed.

\subsubsection{Recapitulation of main features of $\operatorname{ROM~} n^{\circ} 1$ and $n^{\circ} 2$}

Table 1 sums up the main features of both ROM formulations. ROM formulation $n^{\circ} 2$ will be used in the following.

Table 1: Summary of main features of ROM $n^{\circ} 1$ and $R O M n^{\circ} 2$

\begin{tabular}{|c|c|c|}
\hline ROM formulation & $\mathrm{n}^{\circ} 1 \quad$ (cf. 4.2.1) & $\mathrm{n}^{\circ} 2 \quad$ (cf. 4.2.5) \\
\hline Equations taken into & $(1)$-(4) and (5)-(6) & (24)-(27) and (5)-(6) \\
account in formulation & Closure laws (9) and (11) & No closure laws \\
\hline Variables & primitive variables & deviations from initial steady \\
& $\rho, u, e, y, p, T$ & state $\delta \rho, \delta(\rho u), \delta(\rho e), \delta u, \delta p, \delta T$ \\
\hline
\end{tabular}




\begin{tabular}{|c|c|c|}
\hline $\begin{array}{c}\text { Sets of dynamics in } \\
\text { approximations }\end{array}$ & $\begin{array}{c}\text { specific set of dynamics } \\
\text { for each variable }\end{array}$ & $\begin{array}{c}\text { unique set of dynamics for all } \\
\text { variables }\end{array}$ \\
\hline Number of parameters & $5 m^{5}+15 m^{4}+16 m^{3}+$ & $m^{3}+m^{2}+m$ \\
in the dynamical system & $13 m^{2}+7 m$ & Solutions: cf. 4.2.3 \\
\hline Issues / solutions & Issues: cf. 4.2 .2 & \\
\hline
\end{tabular}

\subsection{Input-output data for ROM identification}

The identification of ROM constitutive parameters requires some input-output data, which are a heat power signal $\dot{Q}_{\text {evap }}^{*}(t)$ applied to the evaporator and the corresponding discrete fields of variables $\delta v^{\text {data }}\left(x_{i}, t_{j} ; \dot{Q}_{\text {evap }}^{*}(t)\right), i=1, \ldots, N_{x}, j=$ $1, \ldots, N_{t}^{i d}, v \in\{\rho, \rho u, \rho e, u, p, T\}$, computed with the Finite Volumes detailed model briefly described in section 3.2 .

\subsection{Identification of ROM constitutive parameters}

\subsubsection{Reduction of the number of parameters to be identified}

In order to effectively build a ROM consisting of equations (34) and (38), components of tensors $Q \in \mathbb{R}^{m \times m \times m}, L \in \mathbb{R}^{m \times m}, V \in \mathbb{R}^{m}$ and $H \in \mathbb{R}^{6 N_{x} \times m}$, need to be computed. In the frame of the Modal Identification Method, the analytic form of these tensors is usually not taken into account and their components are identified through an algorithm using optimization techniques. 
In the nonlinear term $\sum_{i=1}^{m} \sum_{j=1}^{m} Q_{k i j} a_{i}(t) a_{j}(t)$ of equation (34), products $a_{i}(t) a_{j}(t)$ are equal to $a_{j}(t) a_{i}(t)$. The low order dynamical system (34) can thus be written under the following form:

$$
\begin{gathered}
\frac{d a_{k}(t)}{d t}+\sum_{i=1}^{m} \sum_{j=i}^{m} Q_{k i j}^{\prime} a_{i}(t) a_{j}(t)+\sum_{i=1}^{m} L_{k i} a_{i}(t)+V_{k} \dot{Q}_{\text {evap }}(t)=0 \\
\forall k=1, \ldots, m
\end{gathered}
$$

The final form of the ROM is thus given by equations (40) and (38).

Let us call $\theta^{\prime}$ the vector gathering components of tensors $Q^{\prime}, L, V$ in equation (40). The number of parameters to be identified for the dynamical system, which was defined up to now by equation (35), can thus be reduced to:

$$
n_{\theta^{\prime}}\left(R O M n^{\circ} 2\right)=m^{2}(m+1) / 2+m^{2}+m
$$

The $n_{H}=6 N_{x} m$ components of matrix $H$ also need to be identified.

\subsubsection{Optimization problem}

The ROM construction is recast into a parameter estimation problem. For a given model order $m$, all parameters to be identified, whose total number is $N_{\text {param }}(m)=$ $n_{\theta^{\prime}}\left(R O M n^{\circ} 2\right)+n_{H}=m\left(m(m+1) / 2+m+1+6 N_{x}\right)$, are identified through the minimization of a functional $\mathcal{J}_{i d}^{(m)}\left(\theta^{\prime}, H\right)$ based on the quadratic deviation between data fields $\delta v^{\text {data }}\left(x_{i}, t_{j} ; \dot{Q}_{\text {evap }}^{*}(t)\right)$ computed with the detailed model (see previous section 4.3) on the one hand and corresponding fields $\delta v^{R O M}\left(x_{i}, t_{j} ; \dot{Q}_{\text {evap }}^{*}, \theta^{\prime}, H\right)$ computed with the ROM (eqs. (40) and (38)) on the other hand, for the same heat power signal $\dot{Q}_{\text {evap }}^{*}(t)$ applied to the evaporator. The quadratic functional $\mathcal{J}_{i d}^{(m)}\left(\theta^{\prime}, H\right)$ thus writes: 


$$
\begin{aligned}
& \mathcal{J}_{\text {id }}^{(m)}\left(\theta^{\prime}, H\right) \\
& =\sum_{\substack{\rho, \rho u, \rho e, u, u \\
p, T}}\left(\frac{\sum_{i=1}^{N_{x}} \sum_{j=1}^{N_{t}^{i d}}\left(\delta v^{R O M}\left(x_{i}, t_{j} ; \dot{Q}_{\text {evap }}^{*}, \theta^{\prime}, H\right)-\delta v^{\text {data }}\left(x_{i}, t_{j} ; \dot{Q}_{\text {evap }}^{*}\right)\right)^{2}}{\sum_{i=1}^{N_{x}} \sum_{j=1}^{N_{t}^{i d}}\left(\delta v^{\text {data }}\left(x_{i}, t_{j} ; \dot{Q}_{\text {evap }}^{*}\right)\right)^{2}}\right)
\end{aligned}
$$

In order to assess the quality of the identified ROM, the mean quadratic relative discrepancy $\varepsilon_{v}^{(m)}$ between data from DM and corresponding values computed by ROM is calculated for each variable $v \in\{\rho, \rho u, \rho e, u, p, T\}$ :

$$
\varepsilon_{v}^{(m)}=\sqrt{\frac{\sum_{i=1}^{N_{x}} \sum_{j=1}^{N_{t}^{i d}}\left(\delta v^{R O M}\left(x_{i}, t_{j} ; \dot{Q}_{\text {evap }}^{*}, \theta^{\prime}, H\right)-\delta v^{\text {data }}\left(x_{i}, t_{j} ; \dot{Q}_{\text {evap }}^{*}\right)\right)^{2}}{\sum_{i=1}^{N_{x}} \sum_{j=1}^{N_{t}^{i d}}\left(\delta v^{\text {data }}\left(x_{i}, t_{j} ; \dot{Q}_{\text {evap }}^{*}\right)\right)^{2}}}
$$

A global quantity $\varepsilon_{g l o b}^{(m)}$ is also defined:

$$
\varepsilon_{\text {glob }}^{(m)}=\sqrt{\frac{\sum_{v} \varepsilon_{v}^{2}}{6}}=\sqrt{\frac{\mathcal{J}_{i d}^{(m)}\left(\theta^{\prime}, H\right)}{6}}
$$

Figure 3 summarizes the identification procedure for a ROM of given order $m$ in the Modal Identification Method. 


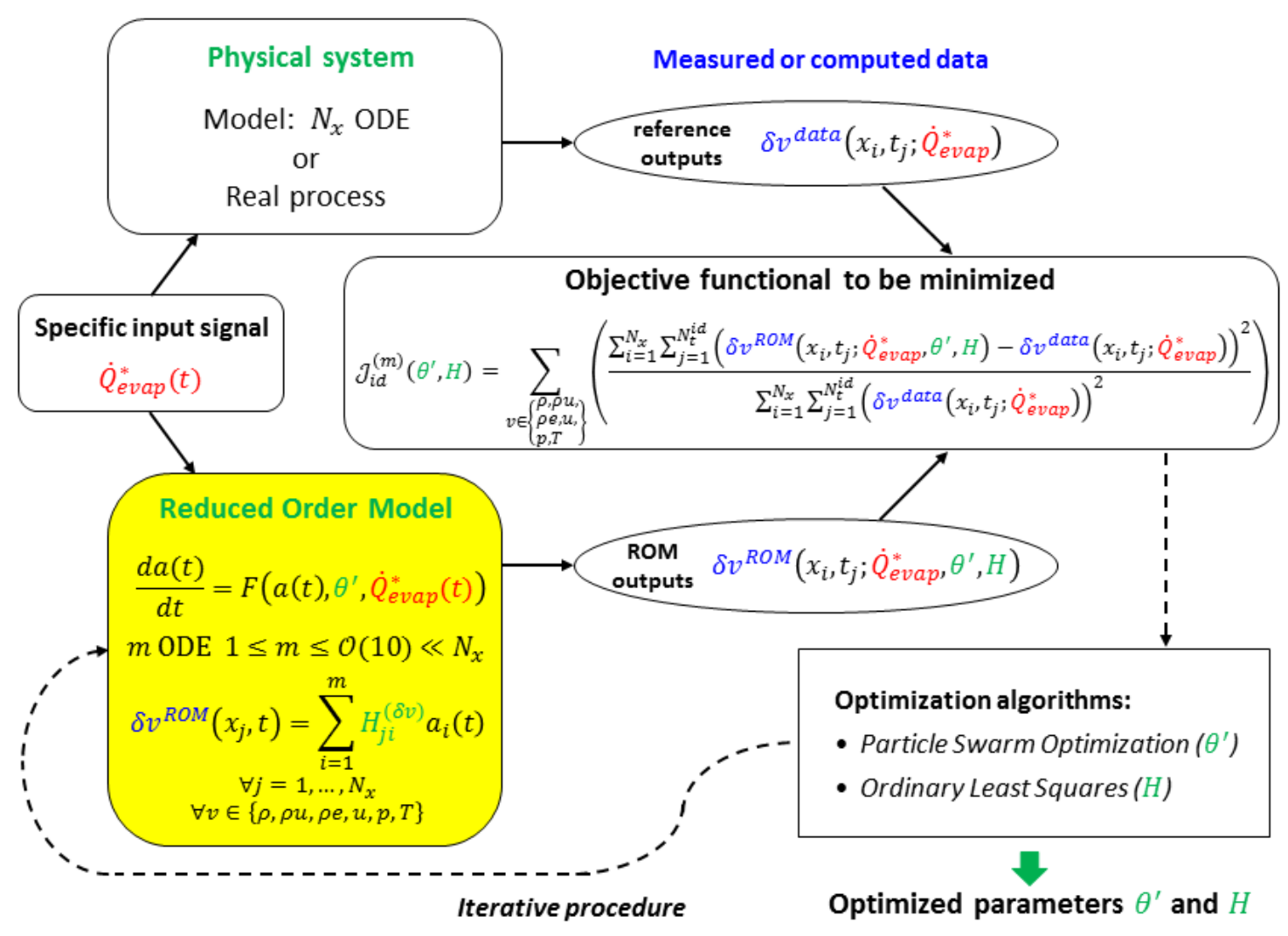

Figure 3 - The Modal Identification Method scheme for order m ROM construction.

\subsubsection{Solving the optimization problem}

First of all $\mathcal{J}_{i d}^{(1)}\left(\theta^{\prime}, H\right)$ is minimized in order to obtain $\theta^{\prime}$ and $H$ associated with a single term in equation (38) and thus defining a ROM of order $m=1$. A ROM of order $m=2$ is then built by minimizing $\mathcal{J}_{i d}^{(2)}\left(\theta^{\prime}, H\right)$ which leads to larger $\theta^{\prime}$ and $H$ associated with two terms in equation (38). ROMs of higher order are then built by incrementing order $m$ and minimizing corresponding functional $\mathcal{J}_{i d}^{(m)}\left(\theta^{\prime}, H\right)$ until a predefined stopping criterion is satisfied. The global procedure is summarized as follows:

1. $m \leftarrow 1$

2. Minimization of $\mathcal{J}_{i d}^{(1)}\left(\theta^{\prime}, H\right) \Rightarrow$ identification of $\theta^{\prime}, H$ for order 1 ROM

3. $m \leftarrow m+1$ 
4. Minimization of $\mathcal{J}_{i d}^{(m+1)}\left(\theta^{\prime}, H\right) \Rightarrow$ identification of new $\theta^{\prime}, H$ for order $m+1$ ROM

5. Test of stopping criterion: 2 possibilities:

5.1 if $\varepsilon_{g l o b}^{(m+1)} \approx \varepsilon_{\text {glob }}^{(m)}$ then STOP else go to 3

5.2 if $\varepsilon_{\text {glob }}^{(m)}$ reach the wished accuracy, then STOP else go to 3

For a given order $m$, a stopping criterion is also used for the minimization of $\mathcal{J}_{i d}^{(m)}\left(\theta^{\prime}, H\right)$. It is usually based on the fact that $\varepsilon_{g l o b}^{(m)}$ does not decrease for a large number of successive iterations.

Equations (40) and (38) show that ROM outputs $\delta v$ have nonlinear dependence on tensors $Q^{\prime}, L, V$ and hence on $\theta^{\prime}$, but depend linearly on $H^{(\delta v)}$. As a consequence, two types of optimization methods are used for the minimization of $\mathcal{J}_{i d}^{(m)}\left(\theta^{\prime}, H\right)$ through a two-steps approach:

1- A nonlinear iterative method is employed for the estimation of $\theta^{\prime}$. An initial guess for $\theta^{\prime}$ is thus required. When identifying the order $m+1 \mathrm{ROM}$, the parameters of the order $m$ ROM previously identified are used as initial guesses for the corresponding unknown parameters in the order $m+1$ ROM. The other parameters arising in the order $m+1 \mathrm{ROM}$ are initially set to zero so that this model gives the same solutions than the order $m$ ROM. The functional $\mathcal{J}_{i d}^{(m+1)}\left(\theta^{\prime}, H\right)$ to be minimized hence starts from the value obtained for $\mathcal{J}_{i d}^{(m)}\left(\theta^{\prime}, H\right)$ and then decreases. A Particle Swarm Optimization (PSO) algorithm [33] has been used in the present work. Details about the home-made PSO code can be found in [26]. A parallelized version has been used for the present work. 
2- At each iteration of the PSO algorithm, parameters in $\theta^{\prime}$ are fixed. The low order state vector $a(t) \in \mathbb{R}^{m}$ is computed at all instants. Matrices $H^{(\delta v)} \in \mathbb{R}^{N_{x} \times m}$, $v \in\{\rho, \rho u, \rho e, u, p, T\}$ are then obtained by minimizing via linear least squares the difference between data $\delta v^{\text {data }}\left(x_{i}, t_{j}\right)$ and ROM outputs $\delta v^{R O M}\left(x_{i}, t_{j}\right)=$ $\sum_{k=1}^{m} H_{i k}^{(\delta v)} a_{k}\left(t_{j}\right), i=1, \ldots, N_{x}, j=1, \ldots, N_{t}^{i d}$ according to (38). The necessary condition $N_{t}^{i d} \geq m$ is fulfilled in practice $\left(N_{t}^{i d}=500\right.$ and $1 \leq m \leq 10$ in the present work). Matrices $H^{(\delta v)}$ are thus computed in a single step at each iteration of the PSO algorithm.

\section{Numerical results}

A series of ROMs taking the form of equations (40) and (38) is now built using the method previously described. The Finite Volumes DM briefly described in section 3.2 is first used with a specific heat power input signal $\dot{Q}_{\text {evap }}^{*}(t)$ to create numerical data fields for the ROMs identification process, and then employed with other inputs signals to generate outputs for testing the identified ROMs.

\subsection{ROMs identification}

The signal $\dot{Q}_{\text {evap }}^{*}(t)$ used to generate data fields for ROMs identification is shown in Figure 4. It covers the [0-1000W] power range corresponding to practical applications of 2PLTs. It is composed of successive rising steps, each one lasting 50 s and hence allowing to reach a new steady state. Although the time step used for the detailed model 
simulations is $\Delta t=10^{-5} \mathrm{~s}$ due to the CFL condition, the time sampling of data used for the ROM identification is made on $N_{t}^{i d}=500$ instants with a time step $\Delta t=1 \mathrm{~s}$.

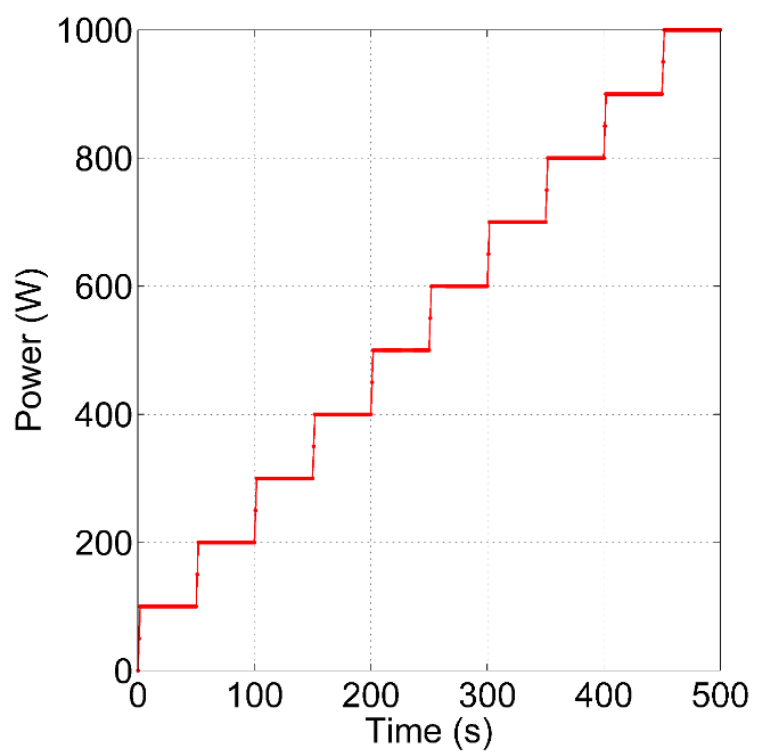

Figure 4 - Input signal $\dot{Q}_{\text {evap }}$ for the identification of the reduced order model parameters.

A series of 5 ROMs (order $m=1$ to 5 ) is built. Solutions of DM and identified ROMs are not shown due to lack of space but values of relative errors $\varepsilon_{v}^{(m)}, v \in\{\rho, \rho u, \rho e, u, p, T\}$ and $\varepsilon_{\text {glob }}^{(m)}$ are gathered in Table 2 . The order 1 ROM corresponds to a single term in equation (38). According to eq.(41), its dynamical system (40) is defined by only 3 parameters which proves to be insufficient for correctly reproducing the data from DM, as shown in Table 2. Incrementing the ROM order yields improvement of ROM quality. The order 5 ROM, which will be called ROM5 in the following, offers very good overall adequacy with DM solutions: $\varepsilon_{\text {glob }}^{(5)}=2.61 \%$. The variable best reproduced is temperature $\left(\varepsilon_{v}^{(5)}=0.10 \%\right)$ whereas the larger error is for mass flow rate $\left(\varepsilon_{v}^{(5)}=5.35 \%\right)$. The CPU time consumption needed for the ROMs identification is given in the last line of Table 2 . It 
took only about $220 \mathrm{~s}$ to build ROM of order 1 . Due to the increasing number of parameters with the model order $m$ (see eq.(41)), the CPU time required also grows quickly with $m$, reaching about $5900 \mathrm{~s}$ for the order 5 ROM. The total CPU time for the identification of ROMs of order 1 to 5 took about $10800 \mathrm{~s}$. This is at least 10 times less than a single computation run of the DM which takes more than $10^{5} \mathrm{~s}$ as stated in $\S 5.2 .3$.

Table 2 - ROM identification. $\varepsilon$ function of the ROM order

\begin{tabular}{|c|c|c|c|c|c|}
\hline ROM order $m$ & 1 & 2 & 3 & 4 & 5 \\
\hline$\varepsilon_{\rho}(\%)$ & 15,49 & 8,60 & 4,39 & 3,07 & 2,12 \\
\hline$\varepsilon_{\rho u}(\%)$ & 23,01 & 21,33 & 6,50 & 6,18 & 5,35 \\
\hline$\varepsilon_{\rho e}(\%)$ & 14,73 & 8,91 & 4,63 & 1,38 & 2,31 \\
\hline$\varepsilon_{u}(\%)$ & 21,75 & 6,76 & 1,27 & 1,22 & 0,93 \\
\hline$\varepsilon_{p}(\%)$ & 29,59 & 2,03 & 1,19 & 1,16 & 1,30 \\
\hline$\varepsilon_{T}(\%)$ & 1,86 & 0,72 & 0,29 & 0,23 & 0,10 \\
\hline$\varepsilon_{g l o b}(\%)$ & 19,74 & 10,48 & 3,79 & 2,96 & 2,61 \\
\hline $\begin{array}{l}\text { CPU Time (s) for } \\
\text { ROM construction }\end{array}$ & 223 & 602 & 1110 & 2934 & 5915 \\
\hline
\end{tabular}

\subsection{ROMs validation tests}

Now that ROMs have been built, the next step consists in testing them with an input heat power signal different from the signal $\dot{Q}_{\text {evap }}^{*}(t)$ used in the identification process. Numerous validation test cases were performed, showing similar results. The input 
signals had different shapes and involved different magnitudes (in the [0-1000W] range) and frequencies. Two of these test cases are presented here. The first one corresponds to a rectangular-shape signal of the thermal load with respect to the time and the second one to sinusoidal variations.

\subsubsection{Case1: rectangular-shape signal}

For this first validation case, the heat power at the evaporator is depicted in Figure 5. Five different levels between $125 \mathrm{~W}$ and $475 \mathrm{~W}$ are applied to the evaporator. The duration of each step is equal to 200s, allowing to reach a steady state for each level. The time step for ROM5 simulation is $\Delta t=1 \mathrm{~s}$. Hence, the signal consists of $N_{t}=1000$ time steps. The aim of this validation case is twofold: i) verify that ROM5 accurately computes the steady states, different from those reached during the identification process, ii) test ROM5 results when the heat power at evaporator suddenly decreases at $t=600 \mathrm{~s}$ although it has been built with a step by step continuously rising signal.

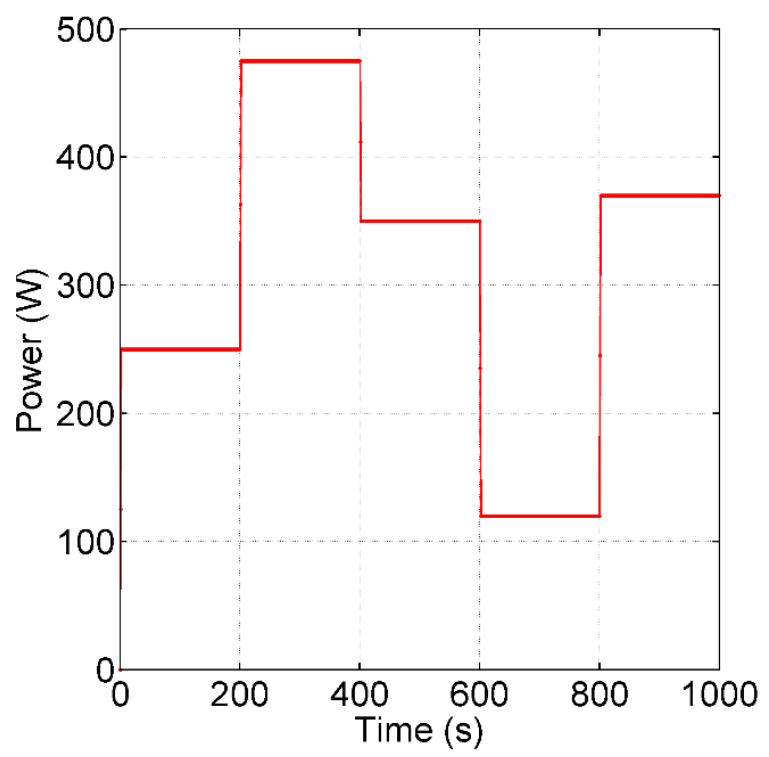

Figure 5 - Input signal $\dot{Q}_{\text {evap }}$ for the validation case 1. 
Table 3 gathers mean quadratic relative errors between data from DM and corresponding values computed by ROMs for each variable, as well as the global relative error. As for the identification process, these errors decrease with ROM order $m$. Let us note that they are nevertheless slightly larger. This is due to the heat power signal form which shows both increasing and decreasing steps of larger magnitude than the identification signal. As shown in Table 3, the errors between ROM5 and DM responses are greater for density, mass flow rate and internal energy per unit volume than for velocity, pressure and temperature.

Table 3 - ROM validation case 1. $\varepsilon$ function of the ROM order

\begin{tabular}{|c|c|c|c|c|c|}
\hline ROM order $m$ & 1 & 2 & 3 & 4 & 5 \\
\hline$\varepsilon_{\rho}(\%)$ & 17.68 & 10.38 & 5.06 & 4.30 & 3.97 \\
\hline$\varepsilon_{\rho u}(\%)$ & 31.11 & 30.12 & 7.83 & 7.64 & 7.49 \\
\hline$\varepsilon_{\rho e}(\%)$ & 17.51 & 11.11 & 5.41 & 4.51 & 4.24 \\
\hline$\varepsilon_{u}(\%)$ & 24.3 & 8.08 & 1.51 & 1.50 & 1.23 \\
\hline$\varepsilon_{p}(\%)$ & 61.61 & 2.52 & 1.69 & 1.61 & 1.48 \\
\hline$\varepsilon_{T}(\%)$ & 2.09 & 0.69 & 0.24 & 0.24 & 0.11 \\
\hline$\varepsilon_{\text {glob }}(\%)$ & 31.56 & 14.20 & 4.50 & 4.13 & 3.95 \\
\hline
\end{tabular}

The space and time variations of density $\rho$, mass flow rate $A \rho u$ and internal energy per unit volume $\rho e$ computed by DM and ROM5, are depicted in Figure 6 by 3D surface plots. For each variable, the same scale is used for DM and ROM5 in order to make the 
comparison easier. The large variations of density and internal energy between the liquid phase and the vapor phase are satisfactorily reproduced by ROM5.

Observing now Figure $6 \mathrm{~b}$, we notice that the $2 \mathrm{PLT}$ 's start-up is faithfully computed by ROM5: the mass flow rate being zero at $t=0 \mathrm{~s}$ stabilizes at different levels with respect to heat power. Furthermore, space and time peaks are smoothed by ROM5.

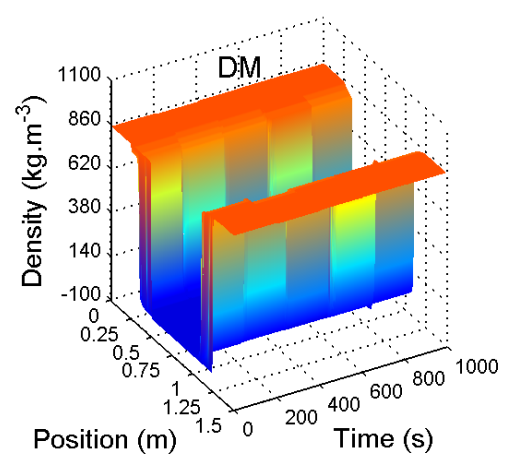

a

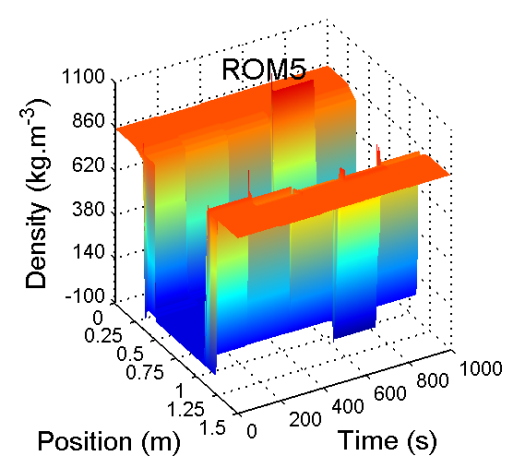

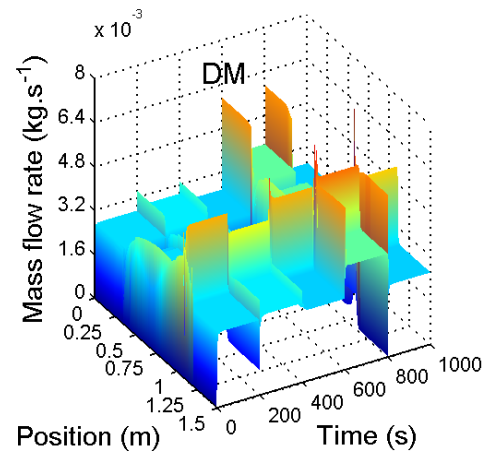

b

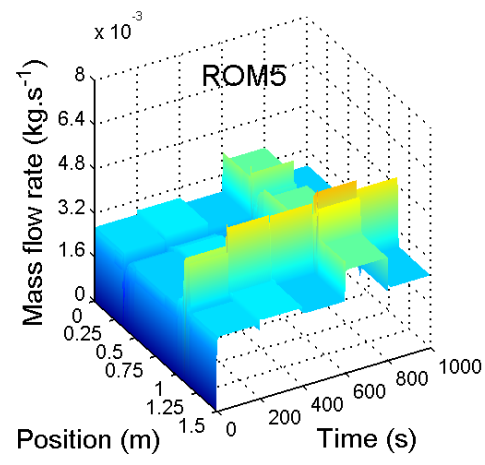

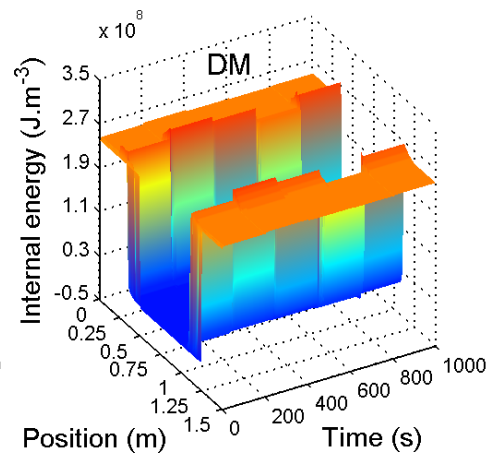

C

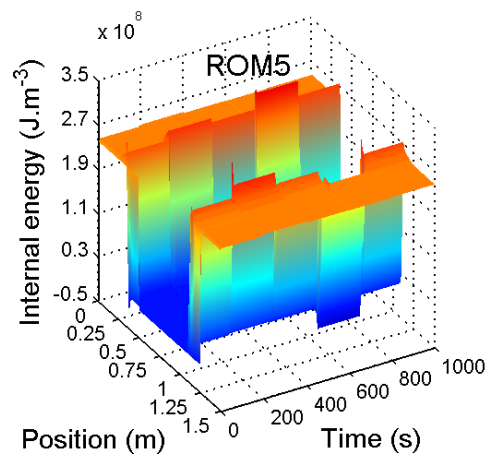

Figure 6-Case 1: space and time map of variables $\rho$, A $\rho u$, $\rho$ e by DM and ROM5.

The space and time evolutions of velocity, pressure and temperature computed by DM and ROM5 are shown in Figure 7. The 2PLT's start-up is accurately calculated by ROM5 although the time variation of the velocity is very sudden, in particular at the evaporator 
outlet: $u=0 \mathrm{~m} \cdot \mathrm{s}^{-1}$ at $t=0 \mathrm{~s}$ to $u \approx 10 \mathrm{~m} \cdot \mathrm{s}^{-1}$ at $t=5 \mathrm{~s}$. Moreover, the velocity discrepancies between the liquid $\left(u \approx 0.01 \mathrm{~m} . \mathrm{s}^{-1}\right)$ and vapor $\left(u \approx 10 \mathrm{~m} . \mathrm{s}^{-1}\right)$ phases are very precisely reproduced. The variations of the pressure are also very accurately described by ROM5, with a relative error $\varepsilon_{p}$ less than $1.5 \%$. Regarding now the temperature variations, the relative error is equal to $0.11 \%$. Temperature is the variable that ROM5 best reproduces.

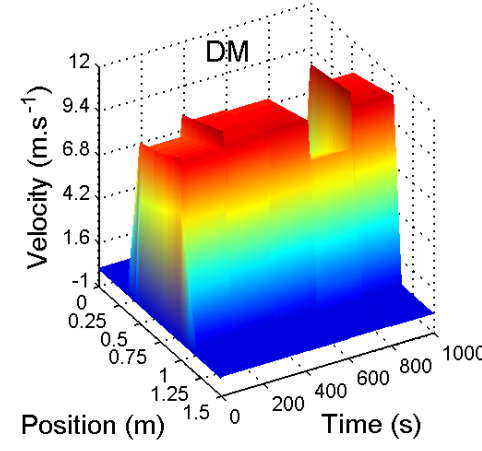

a

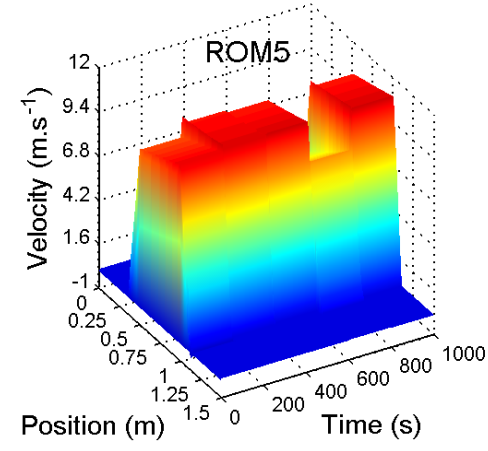

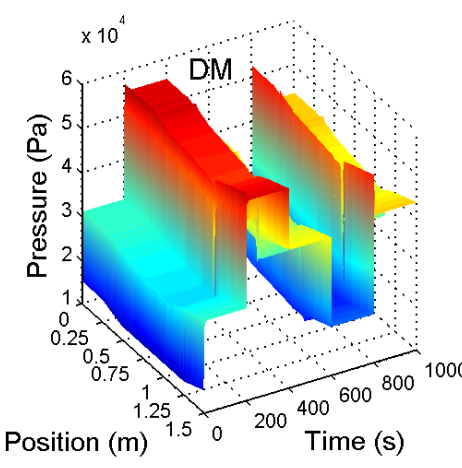

b

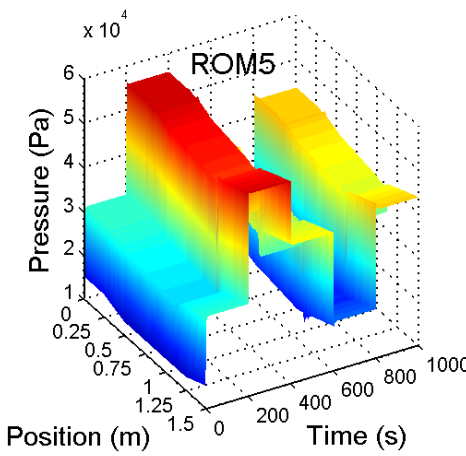

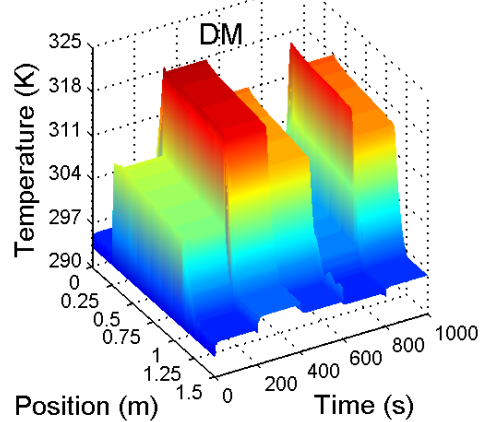

C

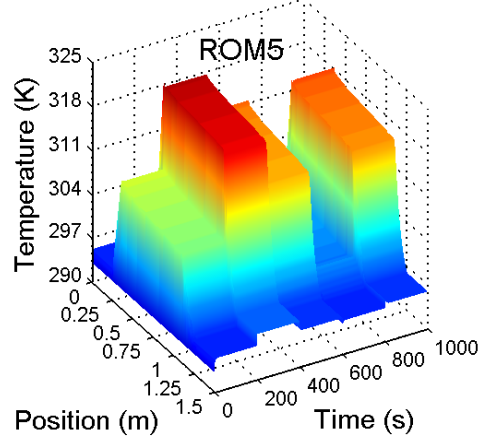

Figure 7 - Case 1: space and time map of variables $u, p, T$ by DM and ROM5.

In order to better analyze the differences between DM and ROM5 responses, the time evolution of the variables $\rho, A \rho u, \rho e, u, p, T$ for two positions along the loop $x=$ 
$0.415 \mathrm{~m}$ (evaporator) and $x=1.165 \mathrm{~m}$ (condenser) are presented in Figure 8 . The legend for all graphs is the one of Figure 8a. The variables evolve globally by steps, showing that for both locations, a steady state is very quickly reached, because the thermal inertia of the duct wall depth of the loop is not taken into account in the DM (and hence in the ROM).

Some deviations appear in the density variations between $t=600 \mathrm{~s}$ and $t=800 \mathrm{~s}$ (Figure 8a) i.e. when heat power decreases. During this period, at these locations, the mixture contains both liquid and vapor, meaning that density takes values in the zones of sharp discontinuities of Figure 6a. These zones are particularly difficult to reproduce by ROMs. A similar behavior can be underlined for mass flow rate (see Figure $8 \mathrm{~b}$ ) and for the internal energy (see Figure 8c). As shown by Figure 8d, the sudden decrease of velocity in the evaporator between $t=600 \mathrm{~s}$ and $t=800 \mathrm{~s}$ is very accurately described by ROM5. However, a velocity difference remains in the condenser. Regarding pressure and temperature, depicted in Figure $8 \mathrm{e}$ and in Figure $8 \mathrm{f}$ respectively, variations are very faithfully reproduced by ROM5. 

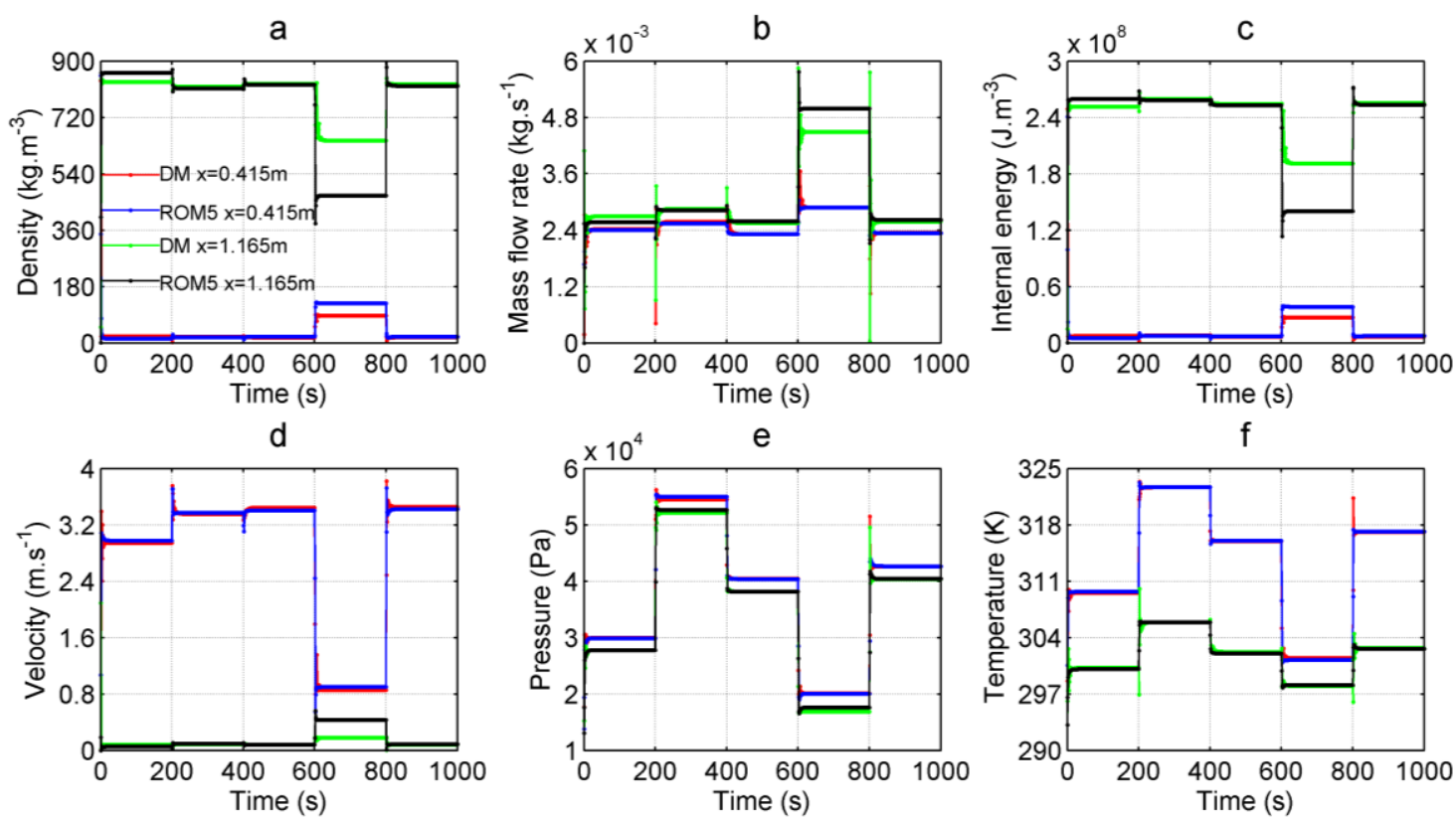

Figure 8 - Case 1: time variations of $\rho, A \rho u, \rho e, u, p, T$ at $x=0.415 m$ (evaporator) and $x=1.165 \mathrm{~m}$ (condenser) by DM and ROM5.

In Figure 9 are shown the space fields of the flow variables at two instants corresponding to the maximum heat power $(t=350 \mathrm{~s})$ and to the minimum heat power $(t=750 \mathrm{~s})$. It can be noticed that the space fields computed by DM and ROM5 are almost superimposed even though the space discontinuities are very large for the variables $\rho, \rho e, u$. 

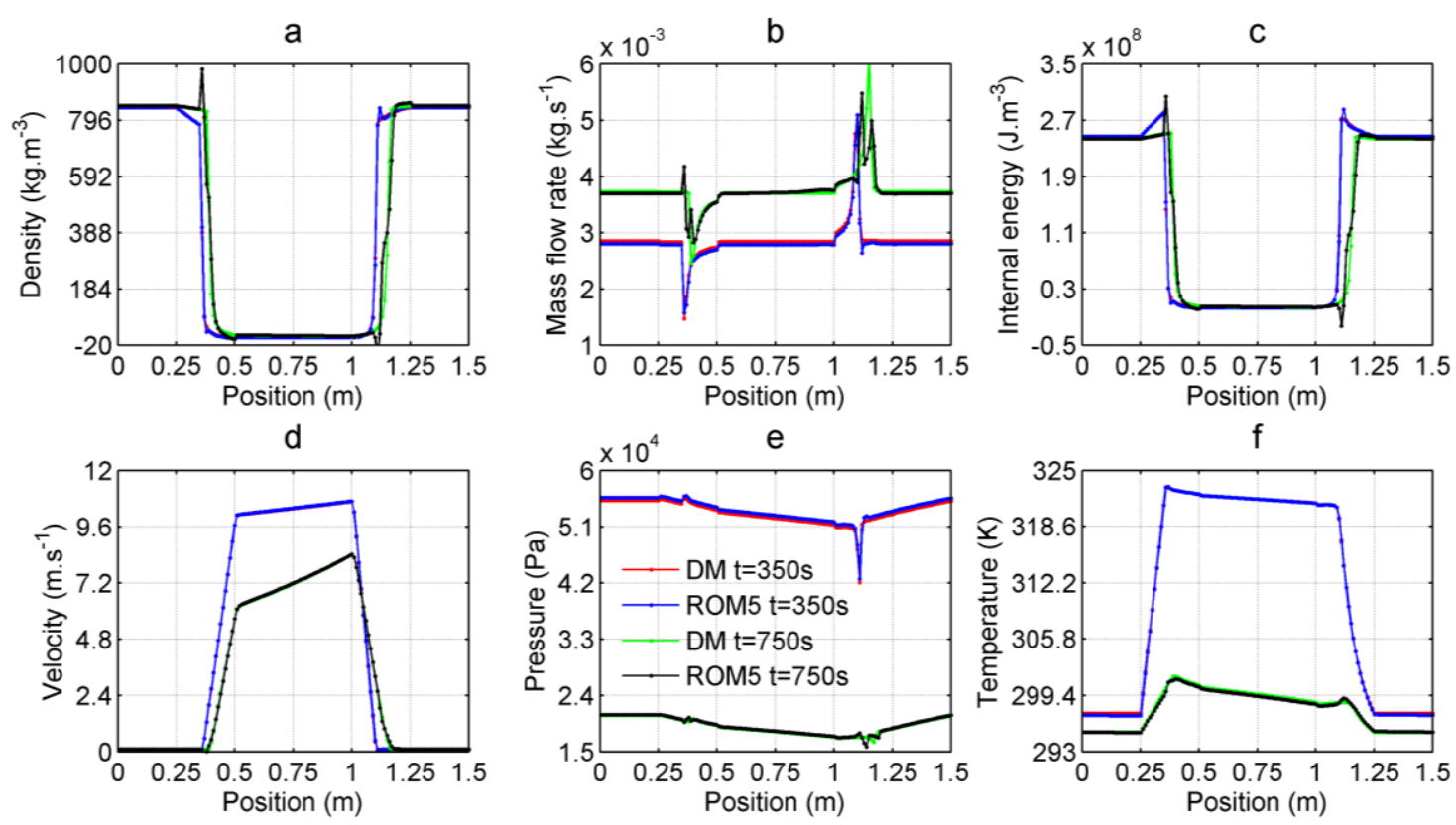

Figure 9 - Case 1: space variations of $\rho, A \rho u, \rho e, u, p, T$ at $t=350 s$ and $t=750 s$ by $D M$ and $R O M 5$.

\subsubsection{Case 2: sinus-shaped signal}

For this second validation case, the thermal load at the evaporator is depicted in Figure 10. The sinusoidal signal whose amplitude and frequency vary evolves slowly in time. Hence, the 2PLT's behavior will not reach a steady state. The time step for ROM5 simulation is $\Delta t=1 \mathrm{~s}$. The signal consists of $N_{t}=2500$ time steps.

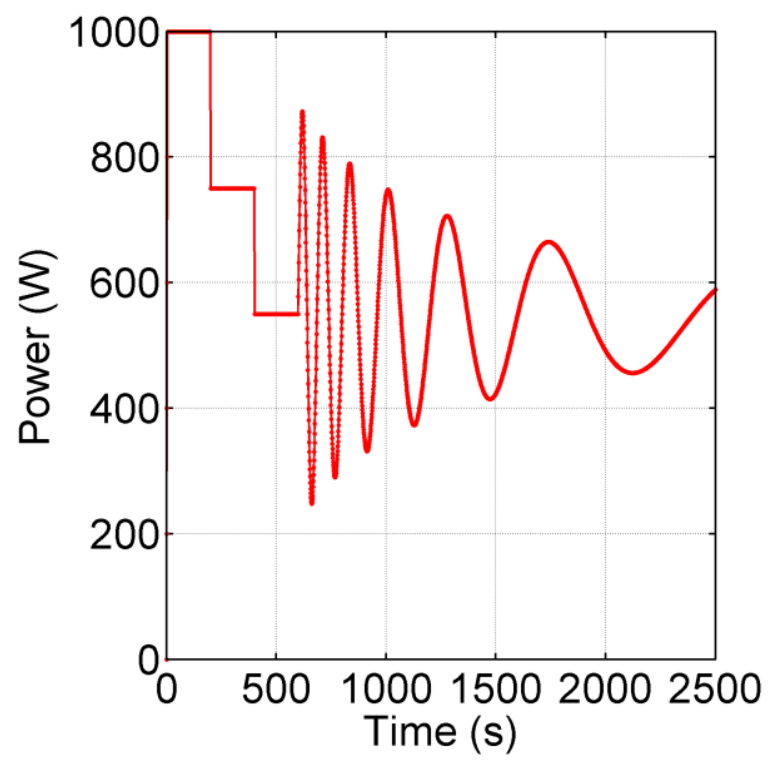


Figure 10 - Input signal $\dot{Q}_{\text {evap }}$ for the validation case 2.

Table 4 summarizes the mean quadratic relative errors between DM and ROMs computed fields for each variable, as well as the global relative error. As for the identification process, these errors decrease with ROM order $m$. Let us note that these errors are slightly smaller. This is due to the heat power signal form which shows smooth variations.

Table 4 - ROM validation case 2. $\varepsilon$ function of the ROM order

\begin{tabular}{|c|c|c|c|c|c|}
\hline ROM order $m$ & 1 & 2 & 3 & 4 & 5 \\
\hline$\varepsilon_{\rho}(\%)$ & 10.04 & 5.73 & 3.35 & 2.80 & 1.80 \\
\hline$\varepsilon_{\rho u}(\%)$ & 21.29 & 16.73 & 6.83 & 6.52 & 4.01 \\
\hline$\varepsilon_{\rho e}(\%)$ & 10.18 & 6.43 & 3.77 & 3.23 & 2.12 \\
\hline$\varepsilon_{u}(\%)$ & 15.06 & 4.60 & 1.10 & 1.12 & 0.81 \\
\hline$\varepsilon_{p}(\%)$ & 20.85 & 2.09 & 1.28 & 1.23 & 1.87 \\
\hline$\varepsilon_{T}(\%)$ & 1.12 & 0.73 & 0.29 & 0.27 & 0.14 \\
\hline$\varepsilon_{g l o b}(\%)$ & 14.84 & 7.96 & 3.54 & 3.26 & 2.16 \\
\hline
\end{tabular}

Time and space variations of the flow variables $\rho, A \rho u, \rho e$ computed by DM and ROM5 are presented in Figure 11 by 3D surface plots. On the whole, variables $\rho$, $\rho$ e present large variations in space due to the high ratio between liquid and vapor densities (around 1000). Apart from some very confined peaks, the space discontinuities are wellreproduced by ROM5. The time evolution of these variables is strongly damped 
compared to the heat power one. The 2PLT start-up is accurately calculated by ROM5, as shown in Figure 11b, where the mass flow rate starts from $0 \mathrm{~kg} . \mathrm{s}^{-1}$, then goes through a maximum before decreasing around $3 \mathrm{~kg} \cdot \mathrm{s}^{-1}$. As shown in Table 4, the error computed for the mass flow rate is the greatest again and reaches $4 \%$. It can be explained by peaks in the evaporator and in the condenser, visible in Figure $11 \mathrm{~b}$ and in Figure 14b. These peaks are confined in time and space and are difficult to reproduce by ROMs.

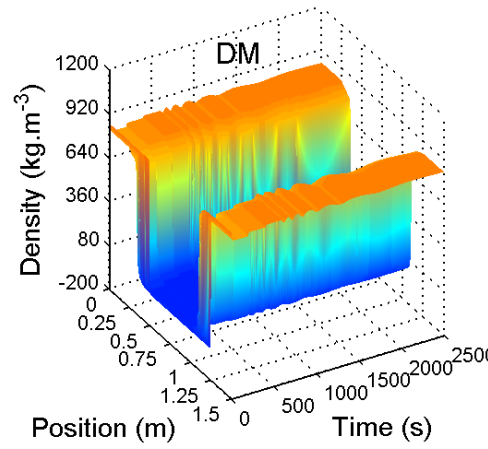

a

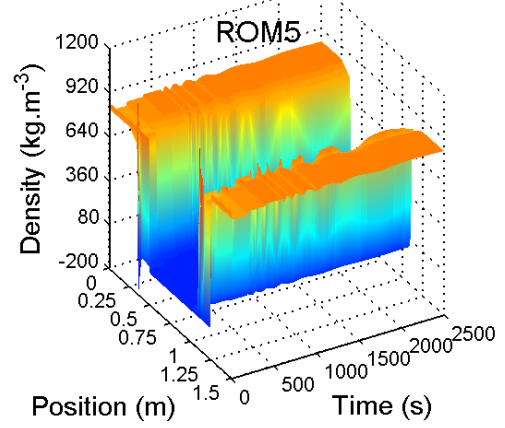

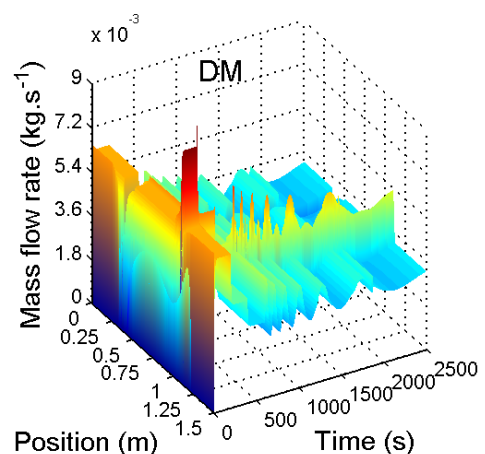

b

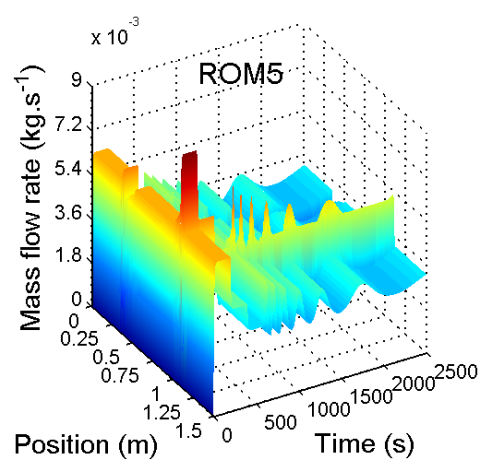

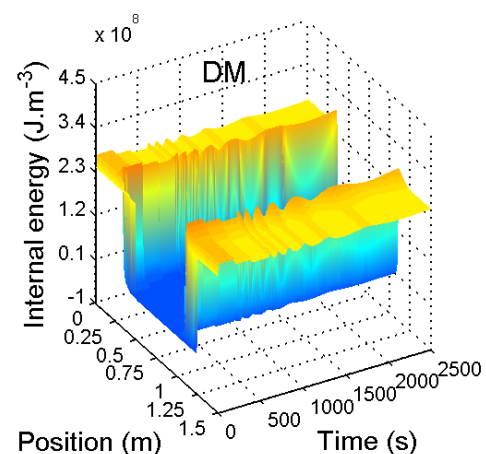

C

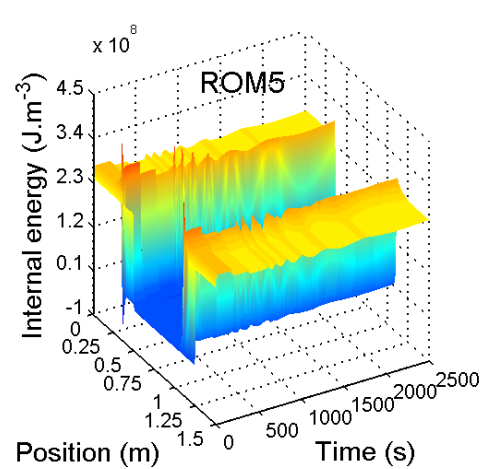

Figure 11 - Case 2: Space and time map of $\rho, A \rho u$, $\rho$ e by DM and ROM5.

The maps for $u, p, T$ are presented in Figure 12. The hard discontinuities of velocity are accurately computed by ROM5 (see Figure $12 \mathrm{a}$ ), this is confirmed by the $0.81 \%$ error 
(see Table 4). Pressure and temperature depicted in Figure $12 \mathrm{~b}$ and Figure $12 \mathrm{c}$ respectively follow the heat power time variations and once again ROM5 provides a good approximation of the DM outputs.

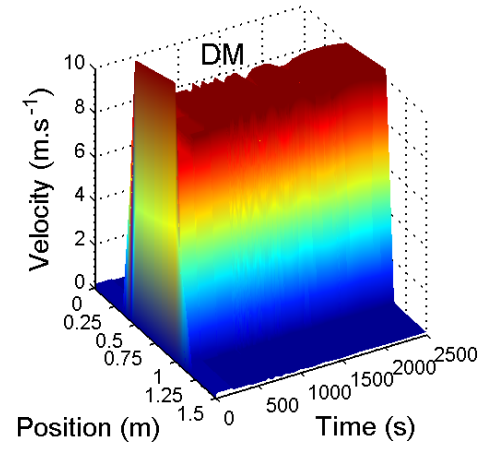

a

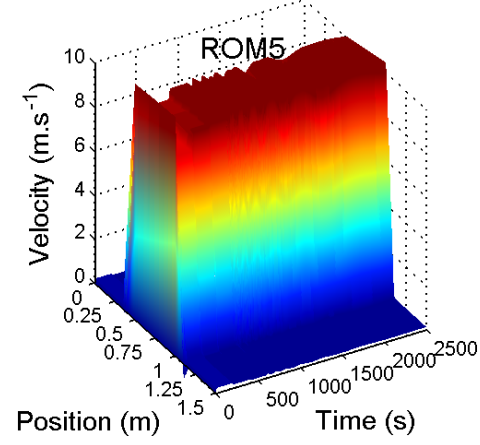

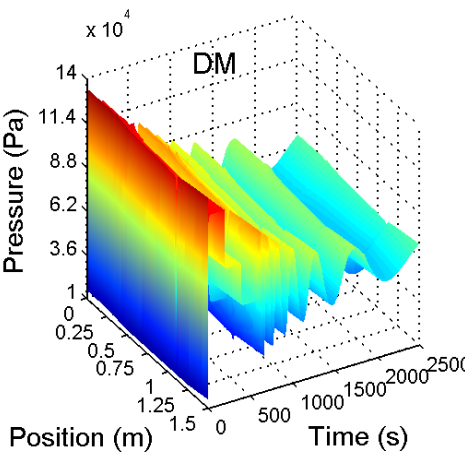

b

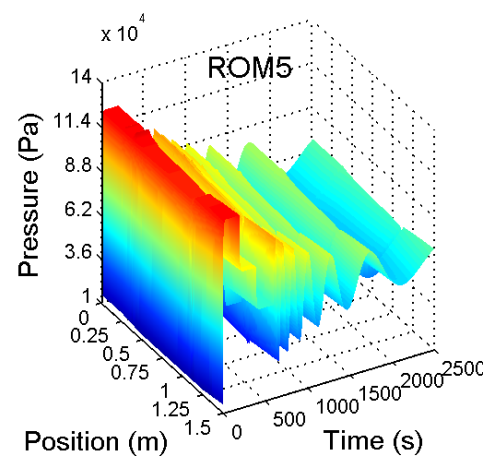

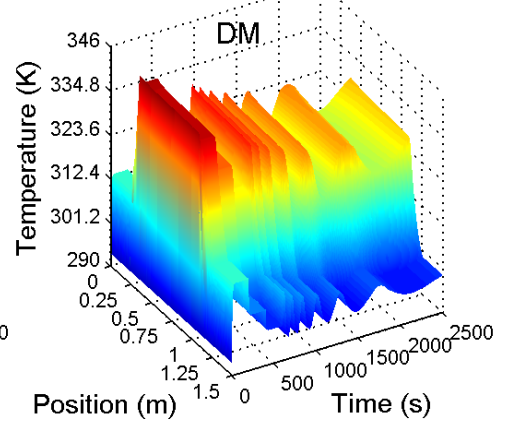

C

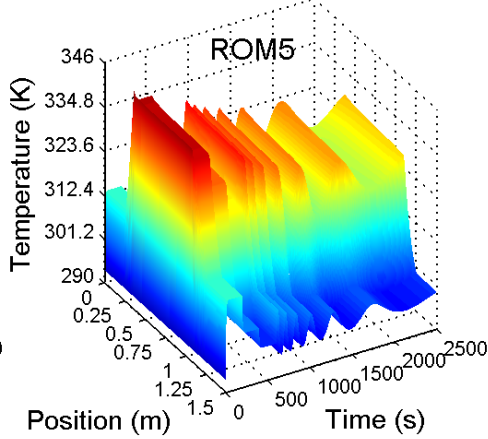

Figure 12 - Case 2: space and time map of $u, p, T$ by DM and ROM5.

Regarding now the time evolution of the variables $\rho, A \rho u, \rho e, u, p, T$ for two positions along the loop $x=0.415 \mathrm{~m}$ (evaporator) and $x=1.165 \mathrm{~m}$ (condenser) depicted in Figure 13 , it can be remarked that the variations with respect to time present the same sinusoidal shape than the heat load applied to the evaporator. These evolutions are slow in time and there is no difficulty for ROM5 to compute these outputs. As usual, the greatest variations between both points are for density (see Figure 13a), mass flow rate 
(see Figure 13b) and velocity (see Figure $13 \mathrm{~d}$ ) because the liquid initially present in the evaporator turns into vapor and the vapor initially present in the condenser becomes liquid.
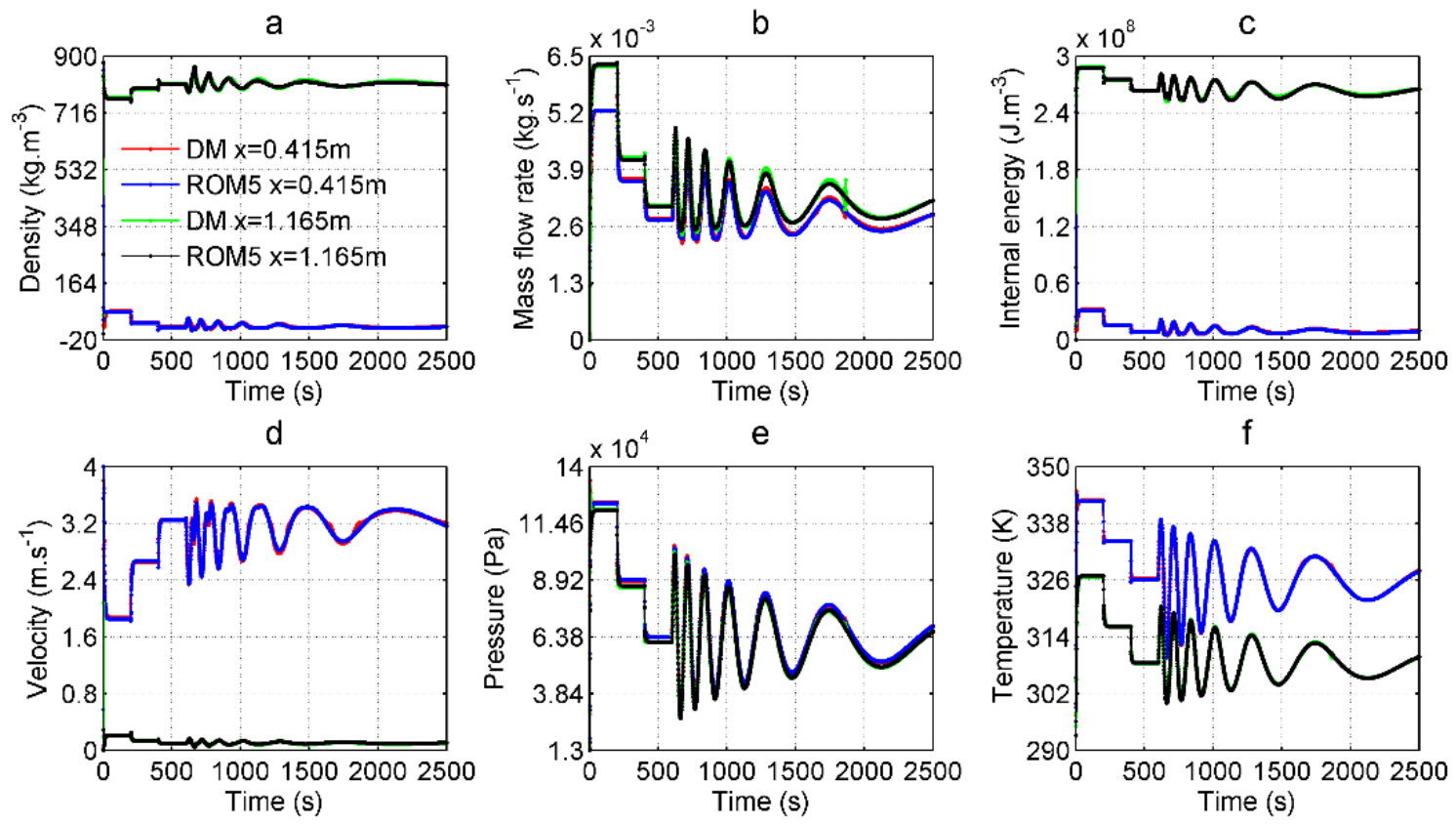

Figure 13 - Case 2: time variations of $\rho, A \rho u, \rho e, u, p, T$ at $x=0.415 \mathrm{~m}$ (evaporator) and $x=1.165 \mathrm{~m}$ (condenser) by DM and ROM5.

Regarding now the space fields of the flow variables at $t=625 \mathrm{~s}$ and $t=1480 \mathrm{~s}$, a very good agreement can be observed between ROM5 and DM outputs, as shown in Figure 14. Of course, this also holds true for other instants of the simulation. Note that for this second test case, the global error is equal to $2.16 \%$ compared to $3.95 \%$ for the first test case. This difference is due to the slow dynamics of the sinusoidal signal. 

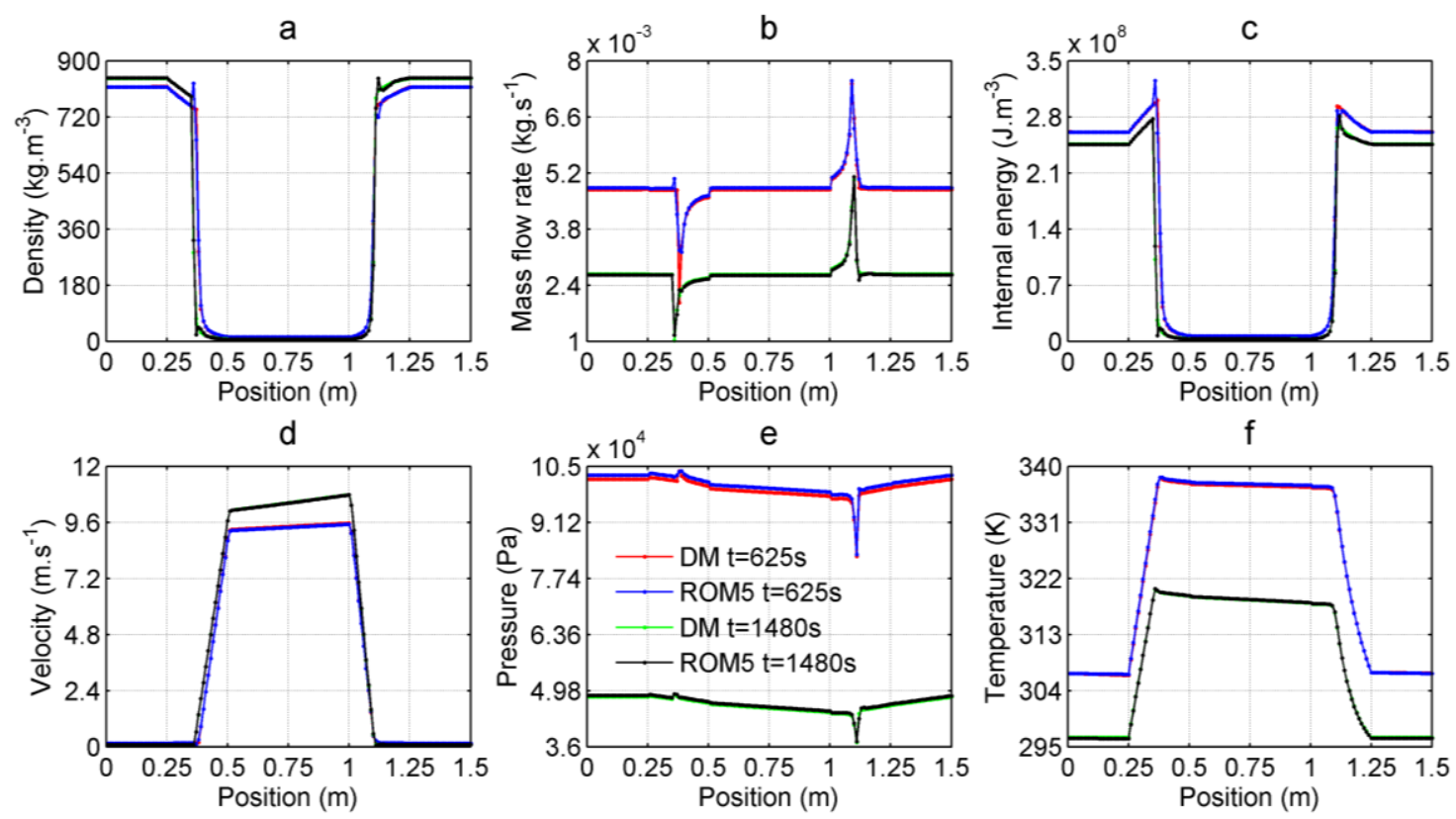

Figure 14 - Case 2: space variations of $\rho, A \rho u, \rho e, u, p, T$ at $t=625 \mathrm{~s}$ and $t=1480 \mathrm{~s}$ by $D M$ and ROM5.

\subsubsection{CPU time gain}

The interest of using a ROM lies in the significant gain in CPU time: $2.4710^{5} \mathrm{~s}$ and 7.29 $10^{5} \mathrm{~s}$ for DM versus $0.02 \mathrm{~s}$ and $0.08 \mathrm{~s}$ for ROM5, for cases 1 and 2 respectively.

This CPU time gain close to $10^{7}$ is due to:

- First, for about a $10^{2}$ factor, the reduction of the number of degrees of freedom of the dynamical system (only 5 for the ROM versus 900 for the DM);

- Second, for about a $10^{5}$ factor, the fact that the reduced model is not constrained by the Courant-Friedrichs-Lewy condition allowing the use of a much larger time step for simulation ( $\Delta t=1 \mathrm{~s}$ for ROM versus $\Delta t=10^{-5} s$ for DM). 


\section{Conclusion}

In this paper, a numerical study is carried out in order to investigate model reduction of a two-phase closed loop thermosyphon. As a detailed model of the 2PLT is needed in order to determine the structure of the reduced order model (ROM), the 1D two-phase flow model describing the liquid-gas mixture in both mechanical and thermal equilibrium is first briefly recalled. Then, from this detailed model, two ROM formulations have been developed via the Galerkin projection method. The first one which takes into account closure laws and uses a specific set of dynamics for each variable, features some drawbacks. The second one which does not take into account closure laws and uses a unique set of dynamics for all variables, is the simplest possible nonlinear ROM. A series of ROMs of order 1 to 5 , based on this second ROM formulation, has been built through a parameter estimation problem. The total CPU time for the identification of ROMs of order 1 to 5 took at least 10 times less than a single computation run of the DM. After the identification stage, two test cases, corresponding to different heat loads at the evaporator, have shown that the overall levels of density, velocity, mass flow rate, pressure, temperature and internal energy in the loop are satisfactorily reproduced by the order 5 ROM with a global relative error less than $5 \%$. The ROM enables a significant gain in CPU time $\left(\approx 10^{7}\right)$. It would be interesting to develop, build and test ROMs of intermediate complexity between ROM $n^{\circ} 1$ and ROM $\mathrm{n}^{\circ} 2$ formulations. Such ROMs with two or three sets of dynamics for describing the six variables could allow improving accuracy while preserving high CPU time gain. Shortly, the ROM will have to take into account the dynamic viscosity $\mu$ as a function of mass fraction and temperature, as it was done for the DM in [14]. Another interesting 
prospect would be the construction of ROMs able to compute fields of variables in response to two independent time-varying inputs: heat power applied to the evaporator and cold external temperature at the condenser (or even mass flow rate of cold secondary fluid driving the heat exchange coefficient). Such ROMs could be employed to investigate feedback control of the 2PLT: temperature regulation of electronics dissipating heat power at evaporator (perturbation) using the heat exchanger input at condenser (actuator). Among other future developments, the identification of low order models directly from measurements recorded on an actual 2PLT (for instance, temperature or pressure measurements at specific locations e.g. at the evaporator), would be of great interest.

\section{Appendix A: the four terms in the Galerkin projection (32)}

The four terms in the Galerkin projection (32) are written below.

$\forall k=1, \ldots, m:$

$$
\begin{gathered}
\int_{0}^{L} R^{(\delta \rho)}(x, t) \phi_{k}^{(\delta \rho)}(x) d x=\sum_{i=1}^{m}\left(M_{1}\right)_{k i} \frac{d a_{i}(t)}{d t}+\sum_{i=1}^{m}\left(L_{1}\right)_{k i} a_{i}(t) \\
\int_{0}^{L} R^{(\delta(\rho u))}(x, t) \phi_{k}^{(\delta(\rho u))}(x) d x \\
=\sum_{i=1}^{m}\left(M_{2}\right)_{k i} \frac{d a_{i}(t)}{d t}+\sum_{i=1}^{m} \sum_{j=1}^{m}\left(Q_{1}\right)_{k i j} a_{i}(t) a_{j}(t) \\
+\sum_{i=1}^{m}\left(\left(L_{2}\right)_{k i}+\left(L_{3}\right)_{k i}+\left(L_{4}\right)_{k i}\right) a_{i}(t)
\end{gathered}
$$




$$
\begin{aligned}
& \int_{0}^{L} R^{(\delta(\rho e))}(x, t) \phi_{k}^{(\delta(\rho e))}(x) d x \\
& =\sum_{i=1}^{m}\left(M_{3}\right)_{k i} \frac{d a_{i}(t)}{d t}+\sum_{i=1}^{m} \sum_{j=1}^{m}\left(\left(Q_{2}\right)_{k i j}+\left(Q_{3}\right)_{k i j}+\left(Q_{4}\right)_{k i j}\right) a_{i}(t) a_{j}(t) \\
& +\sum_{i=1}^{m}\left(\left(L_{5}\right)_{k i}+\left(L_{6}\right)_{k i}\right) a_{i}(t)+\left(V_{0}\right)_{k} \dot{Q}_{e v a p}(t) \\
& \int_{0}^{L} R^{(\delta u)}(x, t) \phi_{k}^{(\delta u)}(x) d x=\sum_{i=1}^{m} \sum_{j=1}^{m}\left(Q_{5}\right)_{k i j} a_{i}(t) a_{j}(t)+\sum_{i=1}^{m}\left(\left(L_{7}\right)_{k i}+\left(L_{8}\right)_{k i}\right) a_{i}(t)
\end{aligned}
$$

Expressions of tensors $M_{i}, i=1, \ldots, 3, L_{i}, i=1, \ldots, 8$ and $Q_{i}, i=1, \ldots, 5$ are given in Appendix B. Equation (33) is obtained by adding the four terms and using the following notations: $M_{0}=\sum_{i=1}^{3} M_{i}, L_{0}=\sum_{i=1}^{8} L_{i}$ and $Q_{0}=\sum_{i=1}^{5} Q_{i}$.

\section{Appendix B: tensors expressions in the Galerkin projection (32)}

In this section, indices $i, j, k$ range from 1 to $m$.

$$
\begin{gathered}
\left(M_{1}\right)_{k i}=\int_{0}^{L} \phi_{i}^{(\delta \rho)}(x) \phi_{k}^{(\delta \rho)}(x) d x \\
\left(L_{1}\right)_{k i}=\int_{0}^{L} \frac{d}{d x}\left(\phi_{i}^{(\delta(\rho u))}(x)\right) \phi_{k}^{(\delta \rho)}(x) d x \\
\left(M_{2}\right)_{k i}=\int_{0}^{L} \phi_{i}^{(\delta(\rho u))}(x) \phi_{k}^{(\delta(\rho u))}(x) d x \\
\left(Q_{1}\right)_{k i j}=\int_{0}^{L} \frac{d}{d x}\left(\phi_{i}^{(\delta(\rho u))}(x) \phi_{j}^{(\delta u)}(x)\right) \phi_{k}^{(\delta(\rho u))}(x) d x
\end{gathered}
$$




$$
\begin{gathered}
\left(L_{2}\right)_{k i}=\int_{0}^{L} \frac{d}{d x}\left(\phi_{i}^{(\delta p)}(x)\right) \phi_{k}^{(\delta(\rho u))}(x) d x \\
\left(L_{3}\right)_{k i}=\frac{64 \mu}{2 d^{2}} \int_{0}^{L} \phi_{i}^{(\delta u)}(x) \phi_{k}^{(\delta(\rho u))}(x) d x \\
\left(L_{4}\right)_{k i}=-g \int_{0}^{L} \epsilon(x) \phi_{i}^{(\delta \rho)}(x) \phi_{k}^{(\delta(\rho u))}(x) d x \\
\left(M_{3}\right)_{k i}=\int_{0}^{L} \phi_{i}^{(\delta(\rho e))}(x) \phi_{k}^{(\delta(\rho e))}(x) d x \\
\left(Q_{2}\right)_{k i j}=\int_{0}^{L} \frac{d}{d x}\left(\phi_{i}^{(\delta(\rho e))}(x) \phi_{j}^{(\delta u)}(x)\right) \phi_{k}^{(\delta(\rho e))}(x) d x \\
\left(Q_{3}\right)_{k i j}=\int_{0}^{L} \phi_{i}^{(\delta p)}(x) \frac{d}{d x}\left(\phi_{j}^{(\delta u)}(x)\right) \phi_{k}^{(\delta(\rho e))}(x) d x \\
\left(Q_{5}\right)_{k i j}=-\int_{0}^{L} \phi_{i}^{(\delta \rho)}(x) \phi_{j}^{(\delta u)}(x) \phi_{k}^{(\delta u)}(x) d x \\
\left.\left.\left(Q_{i}\right)_{k i j}^{(\delta u)}(x)(\rho e)_{0}(x)\right)+p_{0}(x) \frac{d}{d x}\left(\phi_{i}^{(\delta u)}(x)\right)\right) \phi_{k}^{(\delta(\rho e))}(x) d x \\
\left(L_{5}\right)_{k i}=\frac{64 \mu}{2 d^{2}} \int_{0}^{L} \phi_{i}^{(\delta u)}(x) \phi_{j}^{(\delta u)}(x) \phi_{k}^{(\delta(\rho e))}(x) d x \\
\Omega_{c o n d} \phi_{\text {cont }}^{(\delta(\rho e))}(x) d x \\
\text { cond } \phi_{i}^{(\delta T)}(x) \phi_{k}^{(\delta(\rho e))}(x) d x
\end{gathered}
$$




$$
\begin{gathered}
\left(L_{7}\right)_{k i}=\int_{0}^{L} \phi_{i}^{(\delta(\rho u))}(x) \phi_{k}^{(\delta u)}(x) d x \\
\left(L_{8}\right)_{k i}=-\int_{0}^{L} \rho_{0}(x) \phi_{i}^{(\delta u)}(x) \phi_{k}^{(\delta u)}(x) d x
\end{gathered}
$$

\section{Appendix C: proof that matrix $M_{0}$ is invertible}

$$
\begin{aligned}
\left(M_{0}\right)_{k i}=\sum_{j=1}^{3}\left(M_{j}\right)_{k i} & \\
= & \int_{0}^{L}\left(\phi_{i}^{(\delta \rho)}(x) \phi_{k}^{(\delta \rho)}(x)+\phi_{i}^{(\delta(\rho u))}(x) \phi_{k}^{(\delta(\rho u))}(x)\right. \\
& \left.+\phi_{i}^{(\delta(\rho e))}(x) \phi_{k}^{(\delta(\rho e))}(x)\right) d x \quad \forall k \in[1 ; m], \forall i \in[1 ; m]
\end{aligned}
$$

Let us define:

$$
\overrightarrow{\phi_{k}^{\prime}}(x)=\left(\phi_{k}^{(\delta \rho)}(x) \quad \phi_{k}^{(\delta(\rho u))}(x) \quad \phi_{k}^{(\delta(\rho e))}(x)\right)^{T} \quad \forall k=1, \ldots, m
$$

We get $\left(M_{0}\right)_{k i}=\int_{0}^{L} \overrightarrow{\phi_{l}^{\prime}}(x) \cdot \overrightarrow{\phi_{k}^{\prime}}(x) d x \quad \forall k \in[1 ; m], \forall i \in[1 ; m]$

Let us suppose that $M_{0}$ is not invertible. Hence $M_{0}$ would not be of rank $m$ meaning that least one of its rows, let's say raw $\ell$, would be a linear combination of other rows:

$$
\begin{gathered}
\left(M_{0}\right)_{\ell i}=\sum_{\substack{j=1 \\
j \neq \ell}}^{m} \alpha_{j}\left(M_{0}\right)_{j i} \quad \forall i \in[1 ; m] \\
\Leftrightarrow \int_{0}^{L} \overrightarrow{\phi_{l}^{\prime}}(x) \cdot \overrightarrow{\phi_{\ell}^{\prime}}(x) d x=\sum_{\substack{j=1 \\
j \neq \ell}}^{m} \alpha_{j} \int_{0}^{L} \overrightarrow{\phi_{l}^{\prime}}(x) \cdot \overrightarrow{\phi_{j}^{\prime}}(x) d x \quad \forall i \in[1 ; m]
\end{gathered}
$$




$$
\begin{gathered}
\Leftrightarrow \int_{0}^{L} \overrightarrow{\phi_{l}^{\prime}}(x) \cdot\left(\overrightarrow{\phi_{\ell}^{\prime}}(x)-\sum_{\substack{j=1 \\
j \neq \ell}}^{m} \alpha_{j} \overrightarrow{\phi_{J}^{\prime}}(x)\right) d x=0 \quad \forall i \in[1 ; m] \\
\Leftrightarrow \overrightarrow{\phi_{\ell}^{\prime}}(x)=\sum_{\substack{j=1 \\
j \neq \ell}}^{m} \alpha_{j} \overrightarrow{\phi_{j}^{\prime}}(x)
\end{gathered}
$$

Which means: $\left\{\begin{array}{c}\phi_{\ell}^{(\delta \rho)}(x)=\sum_{\substack{j=1 \\ j \neq \ell}}^{m} \alpha_{j} \phi_{j}^{(\delta \rho)}(x) \\ \phi_{\ell}^{(\delta(\rho u))}(x)=\sum_{\substack{j=1 \\ j \neq \ell}}^{m} \alpha_{j} \phi_{j}^{(\delta(\rho u))}(x) \\ \phi_{\ell}^{(\delta(\rho e))}(x)=\sum_{\substack{j=1 \\ j \neq \ell}}^{m} \alpha_{j} \phi_{j}^{(\delta(\rho e))}(x)\end{array}\right.$

Function $\phi_{\ell}^{(\delta \rho)}(x)$ (respectively $\phi_{\ell}^{(\delta(\rho u))}(x)$ and $\phi_{\ell}^{(\delta(\rho e))}(x)$ ) would thus be a linear combination of functions $\phi_{j}^{(\delta \rho)}(x)$ (respectively $\phi_{j}^{(\delta(\rho u))}(x)$ and $\phi_{j}^{(\delta(\rho e))}(x)$ ), $j \in$ $[1 ; m], j \neq \ell$. This is in contradiction with the definition of functions $\phi_{j}^{(\delta \rho)}(x)$, $\phi_{j}^{(\delta(\rho u))}(x)$ and $\phi_{j}^{(\delta(\rho e))}(x), j \in[1 ; m]$ as truncations of bases of the Hilbert space formed by $\mathcal{L}^{2}([0 ; L])$ space equipped with the inner product $(\vec{f}, \vec{g})_{\Omega}=\int_{0}^{L} \vec{f} \vec{g} d x$.

As a consequence, the hypothesis " $M_{0}$ is not invertible" is not valid and $M_{0}$ is invertible. 


\section{Acknowledgements}

This research did not receive any specific grant from funding agencies in the public, commercial, or not-for-profit sectors.

\section{References}

[1] N. Z. Aung and S. Li, «Numerical investigation of riser diameter and inclination on system parameters in a two-phase closed loop thermosyphon solar water heater," Energy Conversion and Management, vol. 75, pp. 25-35, 2013.

[2] J. Li, F. Lin and G. Niu, «An insert-type two-phase closed loop thermosyphon for split-type solar water heaters," Applied Thermal Engineering, vol. 70, pp. 441-450, 2014.

[3] C. C. Chien, C. K. Kung, C. C. Chang, W. S. Lee, C. S. Jwo and S. L. Chen, «Theoretical and experimental investigations of a two-phase thermosyphon solar water heater,» Energy, vol. 36, pp. 415-423, 2011.

[4] T. Yilmaz, «Computer simulation of two-phase flow thermosyphon solar water heating system,» Energy conversion and management, vol. 32, pp. 133-144, 1991.

[5] A. Samba, H. Louahlia-Gualous, S. L. Masson and D. Nörterhäuser, «Two-phase thermosyphon loop for cooling outdoor telecommunication equipments,» Applied Thermal Engineering, vol. 50, pp. 1351-1360, 2013.

[6] C. Sarno, C. Tantolin, R. Hodot, Y. Maydanik and S. Vershinin, «Loop thermosyphon thermal management of the avionics of an in-flight entertainment system," Applied Thermal Engineering, vol. 51, pp. 764-769, 2013. 
[7] P. Zanocco, M. Giménez and D. Delmastro, «Modeling aspects in linear stability analysis of a self-pressurized, natural circulation integral reactor," Nuclear Engineering and Design, vol. 231, pp. 283-301, 2004.

[8] C. P. Marcel, H. F. Furci, D. F. Delmastro and V. P. Masson, «Phenomenology involved in self-pressurized, natural circulation, low thermo-dynamic quality, nuclear reactors: the thermal-hydraulics of the carem-25 reactor," Nuclear Engineering and Design, vol. 254, pp. 218-227, 2013.

[9] C. P. Marcel, F. M. Acuña, D. F. Delmastro and V. P. Masson, «Stability of self-pressurized, natural circulation, low thermo-dynamic quality, nuclear reactors: the stability of performance of the carem-25 reactor, „N Nuclear Engineering and Design, vol. 265, pp. 232243, 2013.

[10] T. E. Tsai, H. H. Wu, C. C. Chang and S. L. Chen, «Two-phase closed thermosyphon vaporchamber system for electronic cooling," International Communications in Heat and Mass Transfer, vol. 37, pp. 484-489, 2010.

[11] C. J. C. Vincent and J. B. W. Kok, «Investigation of the overall transient performance of the industrial two-phase closed loop thermosyphon,» International Journal of Heat and Mass Transfer, vol. 35, pp. 1419-1426, 1992.

[12] H. Bieliński and J. Mikielewicz, «Natural circulation in two phase thermosyphon loop with conventional tubes and minichannels, Heat Transfer - Mathematical Modelling,» Numerical Methods and Information Technology, vol. 19, pp. 475-496, 2011.

[13] W. Qu, «Hydrodynamics of two-phase loop thermosyphon,» Frontiers in Heat Pipes, vol. 1, pp. 1-7, 2010.

[14] S. Bodjona, E. Videcoq, R. Saurel, A. Chinnayya, A. M. Benselama and Y. Bertin, «Transient simulation of a two-phase loop thermosyphon with a model out of thermodynamic 
equilibrium,» International Journal of Heat and Mass Transfer, vol. 108, pp. 2321-2332, 2017.

[15] H. Park and W. Jung, "The Karhunen-Loève Galerkin method for the inverse natural convection problems, „International Journal of Heat and Mass Transfer, vol. 44, pp. 15567, 2001.

[16] N. Hasan and S. Sanghi, «Proper orthogonal decomposition and low-dimensional modelling of thermally driven two-dimensional flow in a horizontal rotating cylinder,» Journal of Fluid Mechanics, vol. 573, pp. 265-295, 2007.

[17] C. Béghein, C. Allery, M. Wacławczyk and J. Pozorski, «Application of POD-based dynamical systems to dispersion and deposition of particles in turbulent channel flow, » International Journal of Multiphase Flow, vol. 58, pp. 97-113, 2014.

[18] R. Bourguet, M. Braza and A. Dervieu, «Reduced-order modeling of transonic flows around an airfoil submitted to small deformations," Journal of Computational Physics, vol. 230, pp. 159-184, 2011.

[19] I. Kalashnikova, B. van Bloemen Waanders, S. Arunajatesan and M. Barone, «Stabilization of projection-based reduced order models for linear time-invariant systems via optimization-based eigenvalue reassignment," Computer Methods in Applied Mechanics and Engineering, vol. 272, pp. 251-270, 2014.

[20] A. Quarteroni, G. Rozza and A. Manzoni, «Certified reduced basis approximation for parametrized partial differential equations and applications," Journal of Mathematics in Industry, vol. 1:3, 2011

[21] D. Knezevic and J. Peterson, «A high-performance parallel implementation of the certified reduced basis method,» Computer Methods in Applied Mechanics and Engineering, vol. 200, pp. 1455-1466, 2011. 
[22] T. Bui-Thanh, K. Willcox, O. Ghattas and B. van Bloemen Waanders, «Goal-oriented, modelconstrained optimization for reduction of large-scale systems, " Journal of Computational Physics, vol. 224, n%12, p. 880-896, 2007.

[23] M. Aghighi, A. Ammara, C. Metivier, M. Normandin and F. Chinesta, «Non-incremental transient solution of the Rayleigh-Bénard convection model by using the PGD, » Journal of Non-Newtonian Fluid Mechanics, vol. 200, pp. 65-78, 2013.

[24] O. Balima, Y. Favennec, M. Girault and D. Petit, "Comparison between the modal identification method and the POD-Galerkin method for model reduction in nonlinear diffusive systems," International Journal for Numerical Methods in Engineering, vol. 67, pp. 895-915, 2006.

[25] M. Girault, E. Videcoq and D. Petit, «Estimation of time-varying heat sources through inversion of a low order model built with the modal identification method from in-situ temperature measurements, "International Journal of Heat and Mass Transfer, vol. 53, n%11-3, pp. 206-219, 2010.

[26] Y. Rouizi, M. Girault, Y. Favennec and D. Petit, «Model reduction by the Modal Identification Method in forced convection: application to a heated flow over a backwardfacing step, » International Journal of Thermal Sciences, vol. 49, pp. 1354-1368, 2010.

[27] L. Cordier, M. Girault and D. Petit, «Reduced Order Modeling by Modal Identification Method and POD-Galerkin approach of the heated circular cylinder wake in mixed convection, „ Journal of Physics: Conference Series, vol. 395(1) 012102, 2012.

[28] M. Girault and E. Videcoq, «Temperature regulation and tracking in a MIMO system with a mobile heat source by LQG control with a low order model,» Control Engineering Practice, n%121, pp. 333-349, 2013. 
[29] K. Bouderbala, H. Nouira, M. Girault and E. Videcoq, «Experimental thermal regulation of an ultra-high precision metrology system by combining Modal Identification Method and Model Predictive Control,» Applied Thermal Engineering, vol. 104, pp. 504-515, 2016.

[30] O. Le Metayer, J. Massoni and R. Saurel, «Elaborating equations of state of a liquid and its vapor for two-phase flow models," International Journal of Thermal Sciences, vol. 43, p. 265-276, 2004.

[31] R. Goodwin, «Methanol thermodynamic properties from 176 to $673 \mathrm{~K}$ at pressures to 700 bar," Journal of Physical and Chemical Reference Data, vol. 16, p. 888-896, 1987.

[32] A. Jafarian and A. Pishevar, "An exact multiphase Riemann solver for compressible cavitating flows, » International Journal of Multiphase Flow, vol. 88, pp. 152-166, 2017.

[33] M. Clerc, Particle Swarm Optimization, New-York: Wiley-ISTE, 2013. 